\title{
Nanoenergetics by Plasma Processing
}

\section{PennState}

College of Engineering

CHEMICAL ENGINEERING

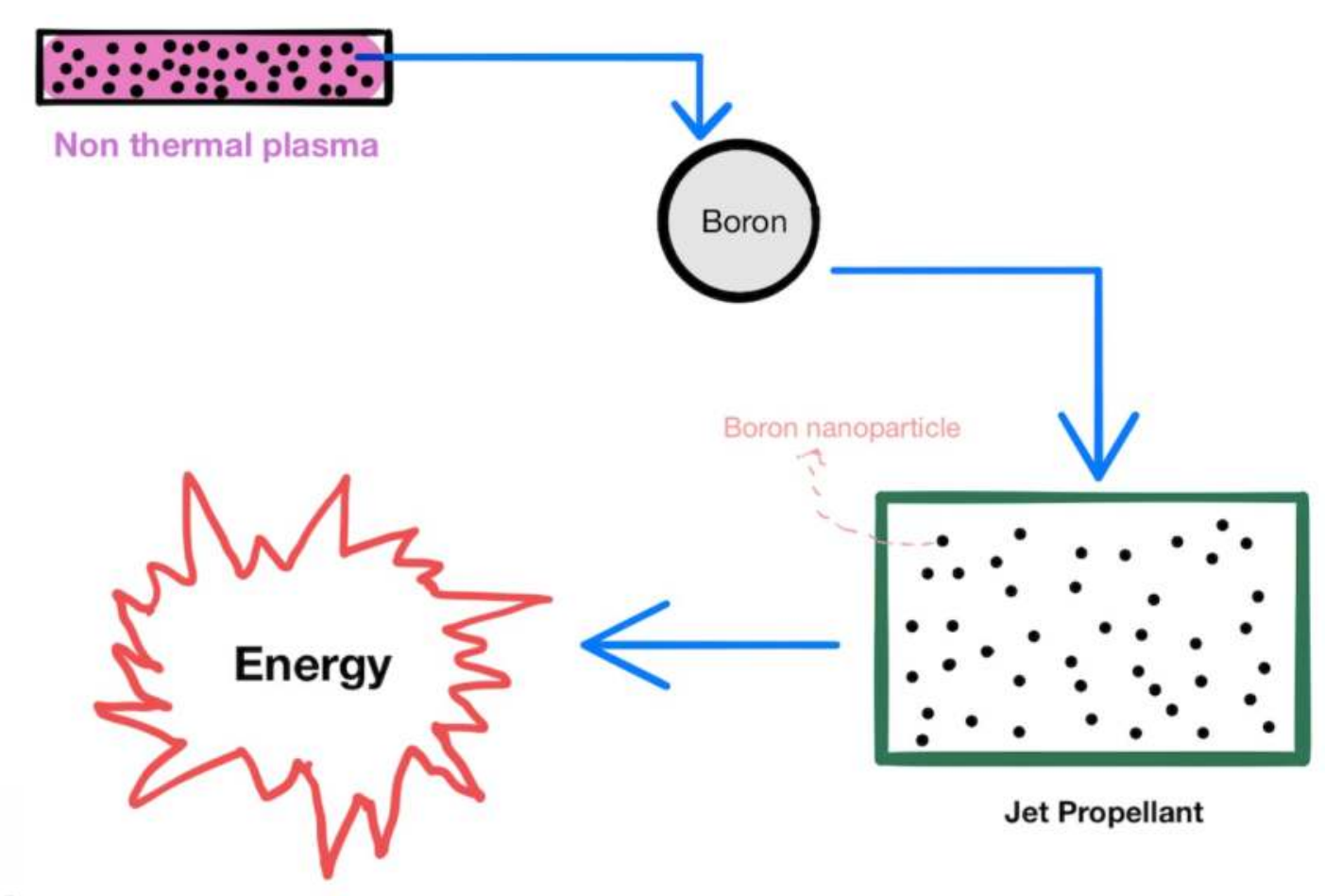

Advisor: Dr. Themis Matsoukas

March, 25, 2020 


\section{Motivation}

Jet Propellants

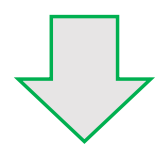

Volumetric Enthalpy of Combustion: $34 \mathrm{KJ} / \mathrm{cc}$

How to extract more energy in volume limited propulsion systems?

\section{Adding solid additives}

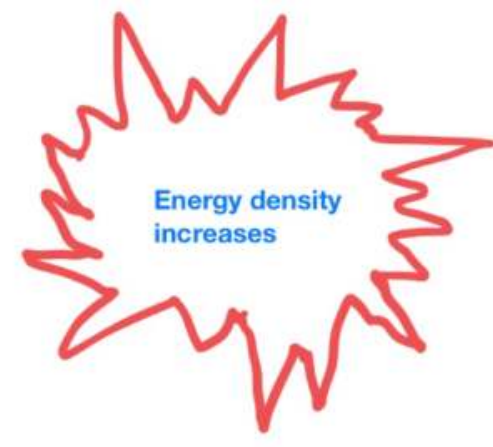

Solid additives (low concentrations)

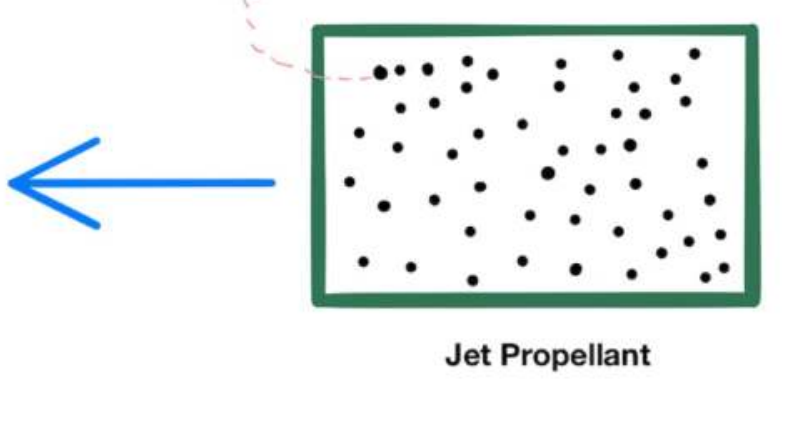

घravimetric heating value $(\mathrm{KJ} / \mathrm{g})=$ Volumetric heating value $(\mathrm{KJ} / \mathrm{cc})$

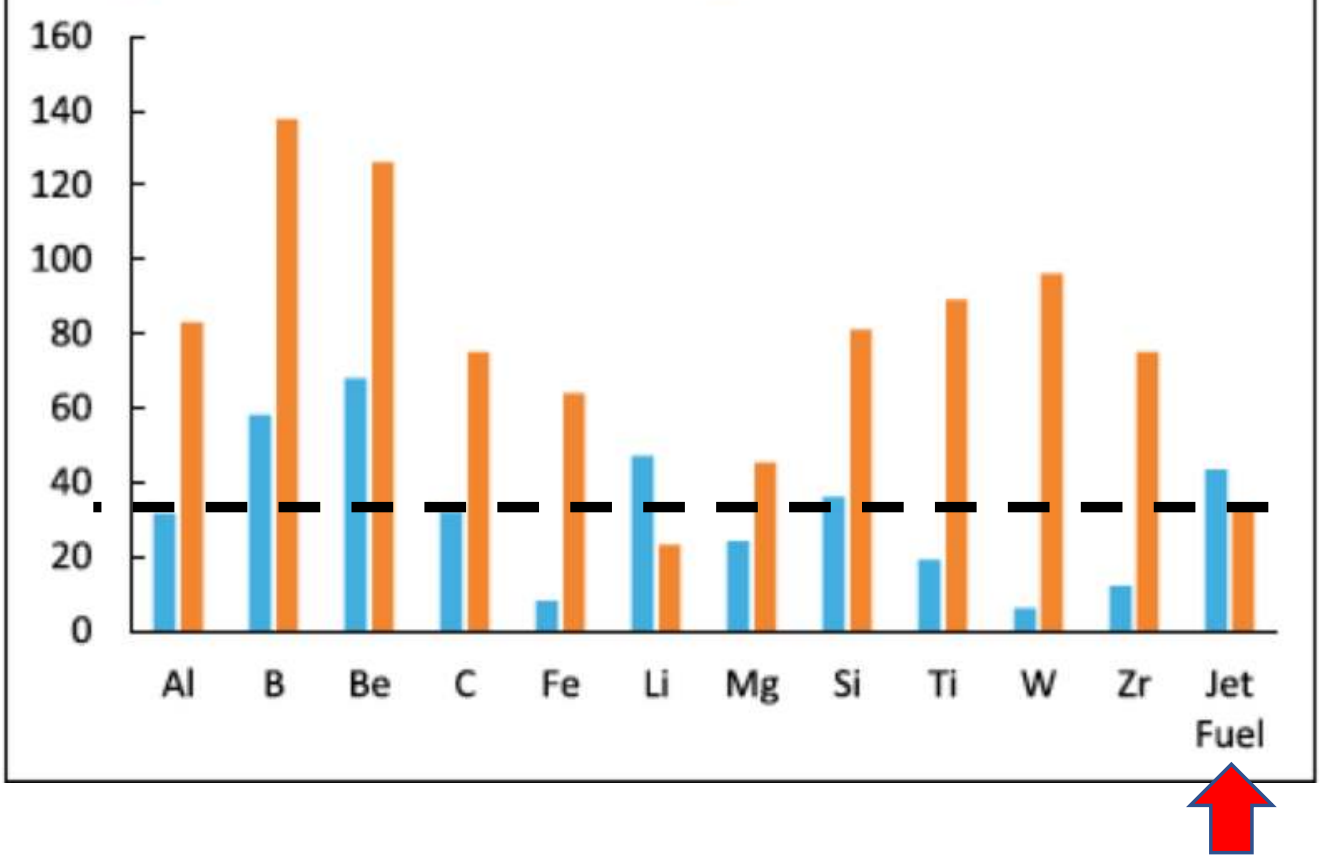

Ojha et al. Progress in Aerospace Sciences 2018,100, 18-45. 


\section{Nanometer sized additives have distinct advantages}
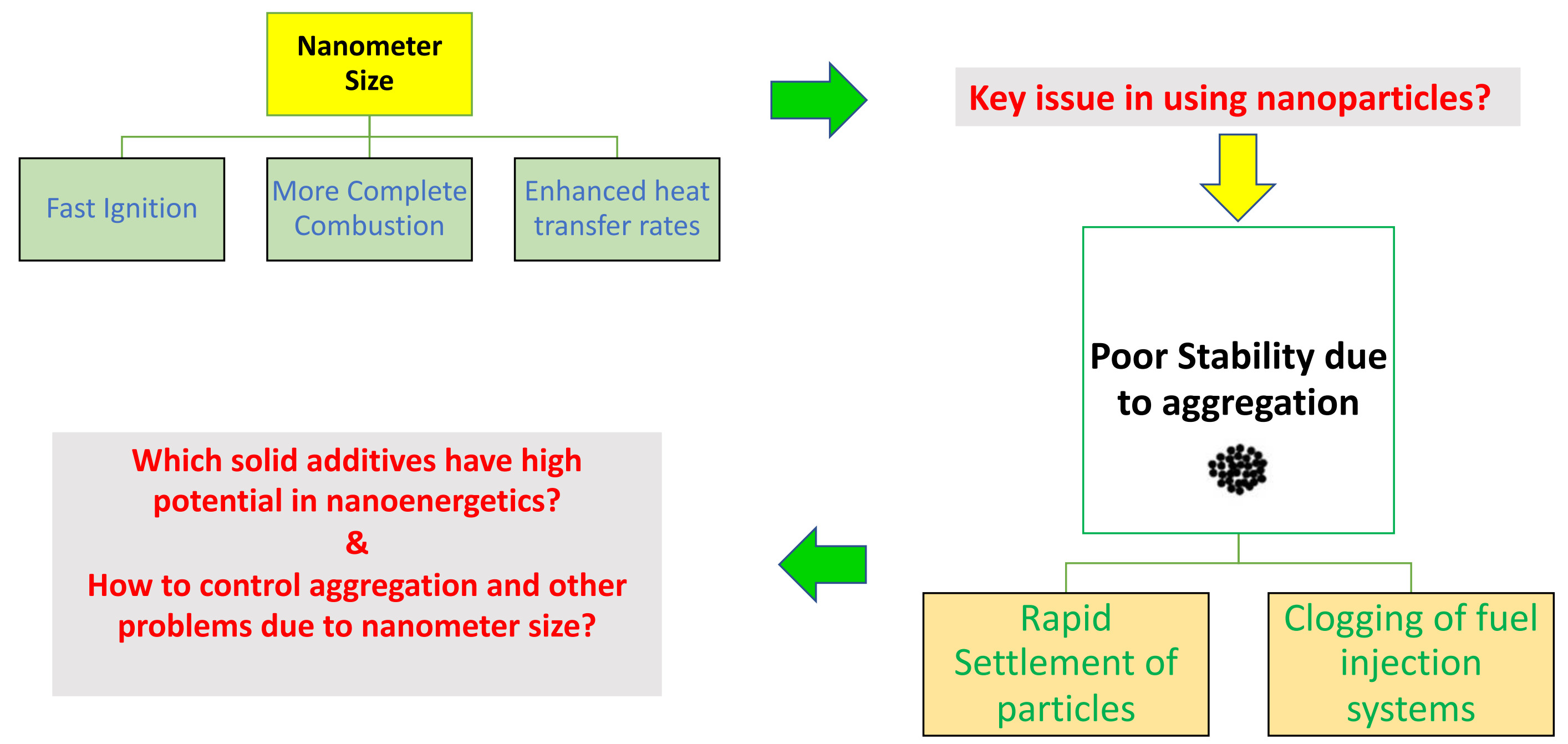


\section{Boron nanoparticles (BNP) have drawn maximum attention of researchers to be used as additives}

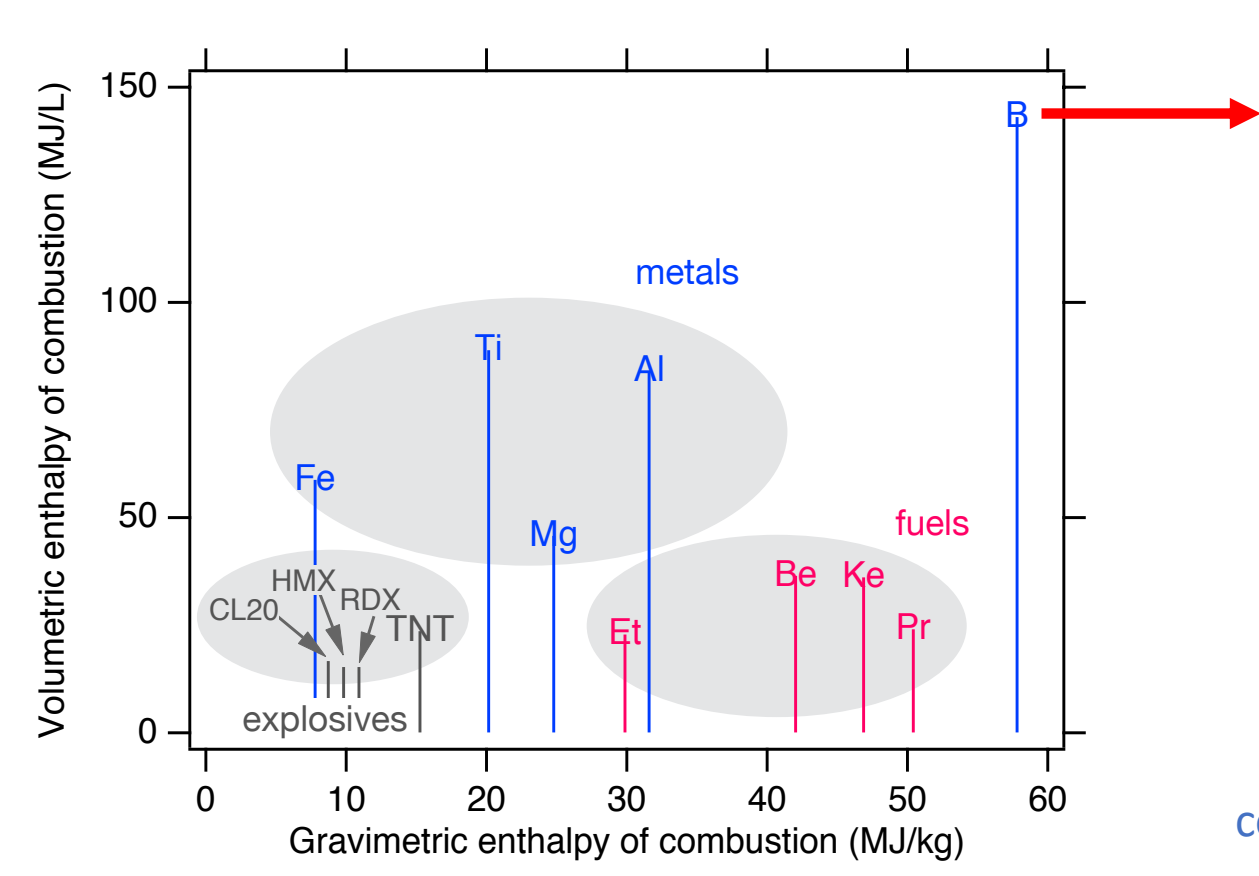

Dreizin E.L., Progress in engineering and Combustion Science, Vol 35 Issue 2, Pages 141-167, 2009
Highest volumetric \& gravimetric enthalpy of combustion $(140 \mathrm{KJ} / \mathrm{cc}, 58 \mathrm{KJ} / \mathrm{g})$

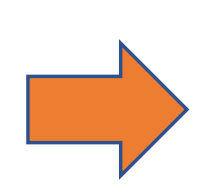

Good choice for volume limited combustion systems
Volumetric combustion enthalpy of ethanol (KJ/cc)

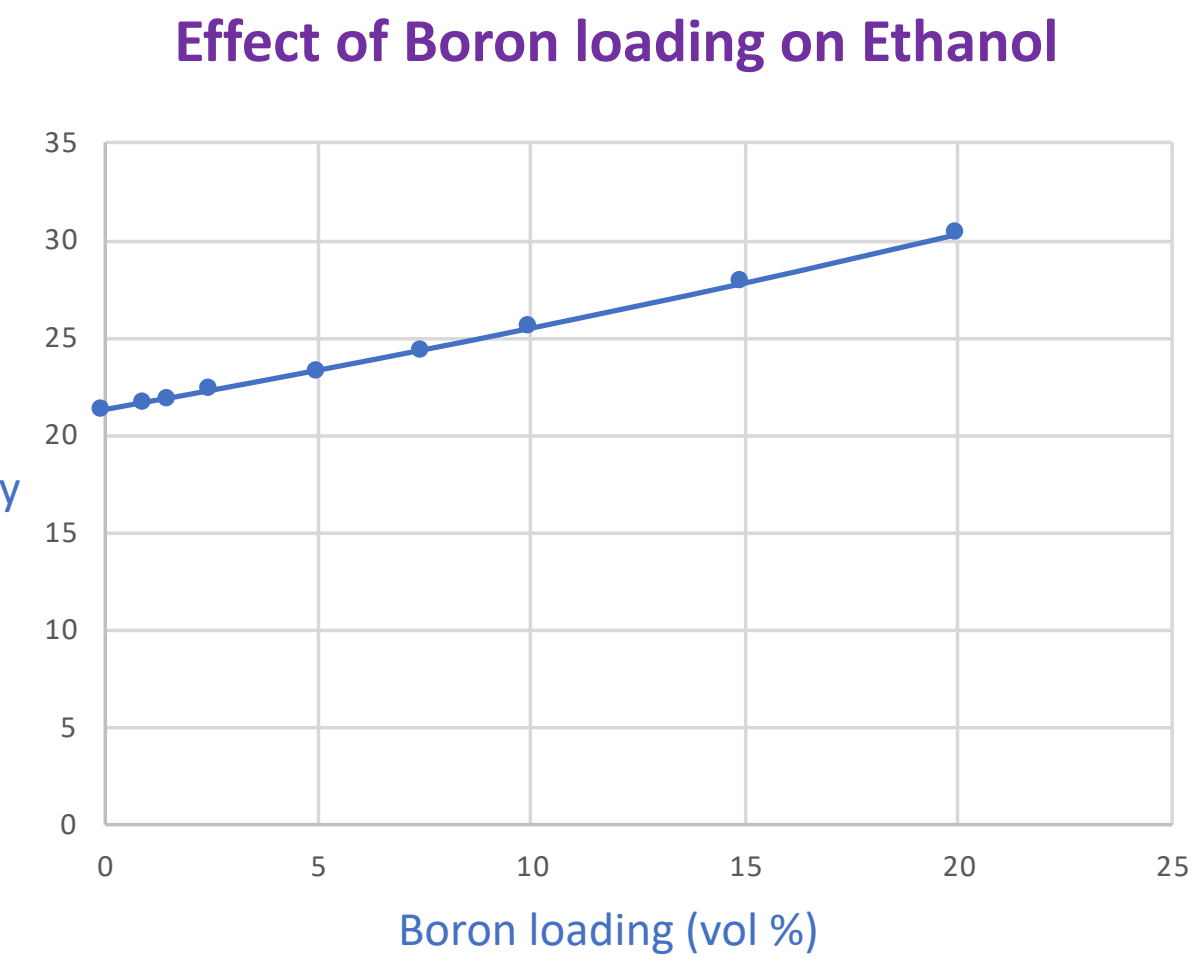

$10 \%$ loading

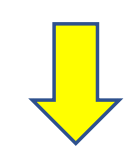

$+19 \%$ in Energy density 


\section{Energetic potential of B can be harnessed in nanoparticulate}

form. But some roadblocks are there!

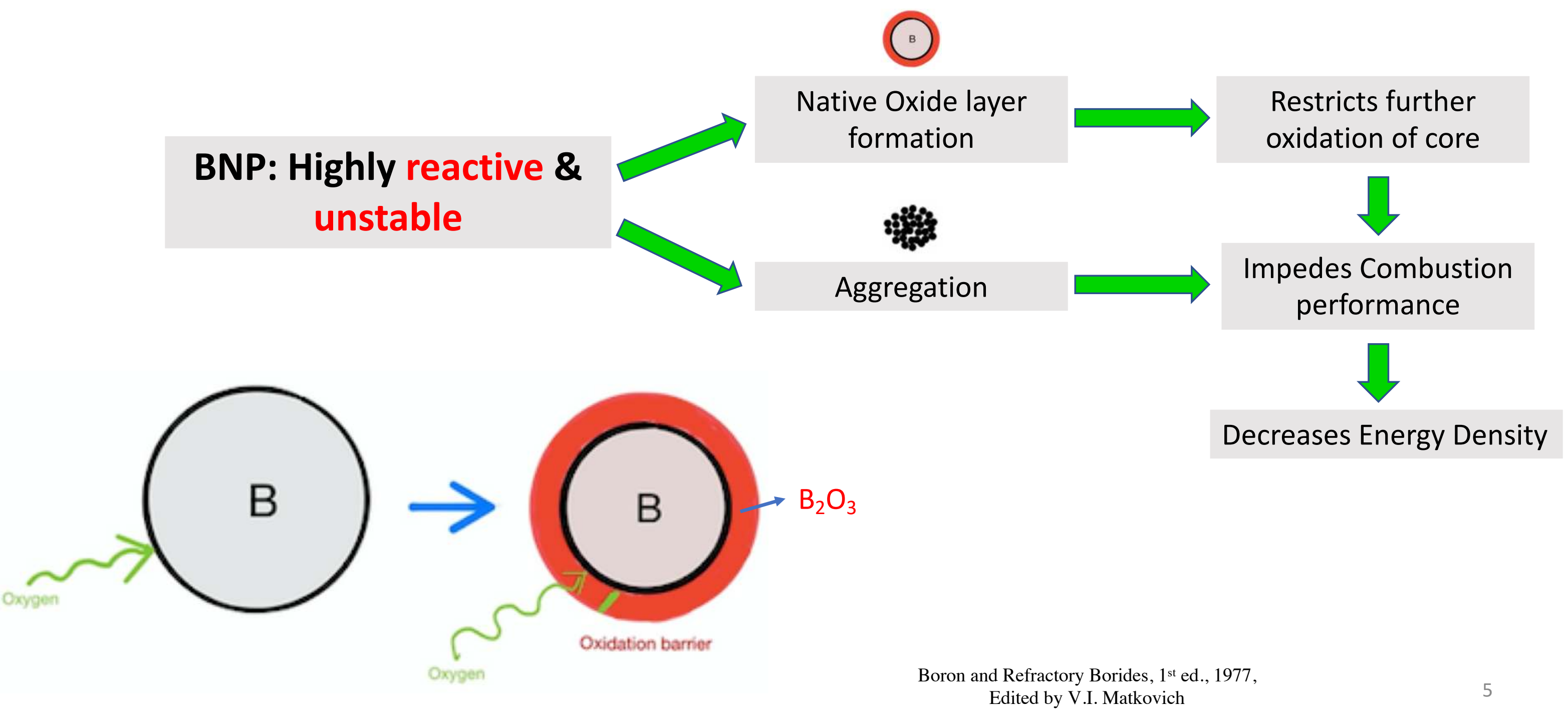




\section{Various reduction methods are available in literature}
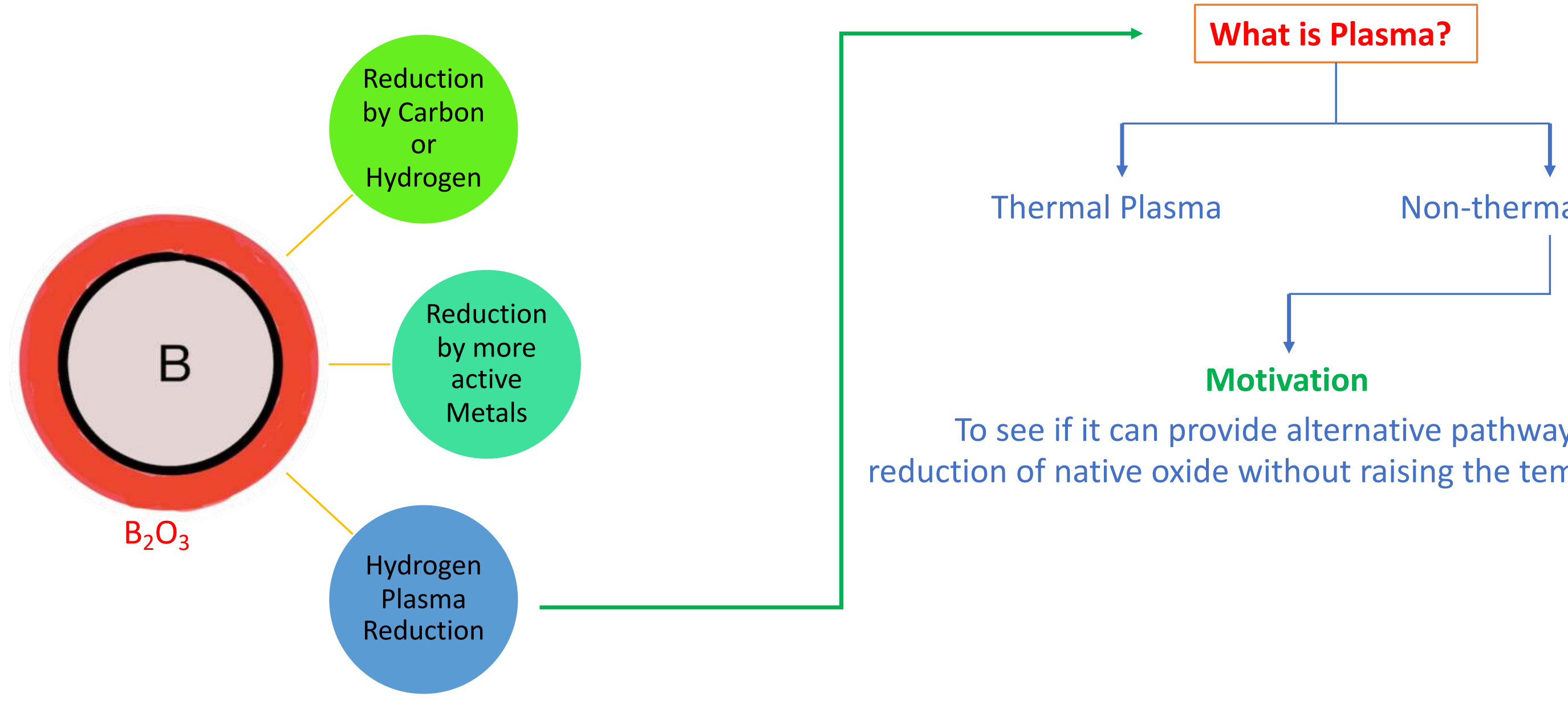

Thermal Plasma

Non-thermal Plasma

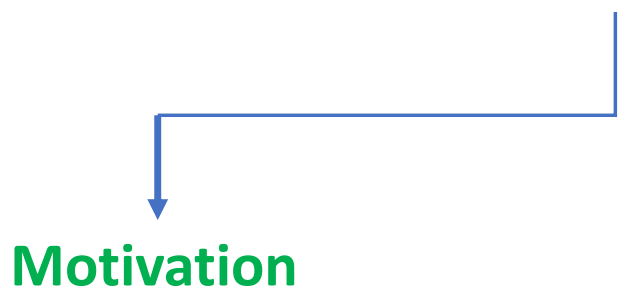

To see if it can provide alternative pathway for reduction of native oxide without raising the temperature? 


\section{Novelty - Non-thermal hydrogen plasma (HP) can be used to reduce native oxide layer from BNP}

\section{Previous work/Literature} Reduction of native oxide

from $\mathrm{Cu}, \mathrm{Ru}, \mathrm{Ge}$, Si surfaces
Thermodynamic Feasibility

At low temperatures

Driving Force of Reduction

Highly reactive hydrogen atoms, ions \& vibrationally excited $\mathrm{H}_{2}$ molecules

\section{Advantages}

- Dry process. Does Not involve many chemicals and post processing separations

- By-product - Water vapor, not an environmental threat

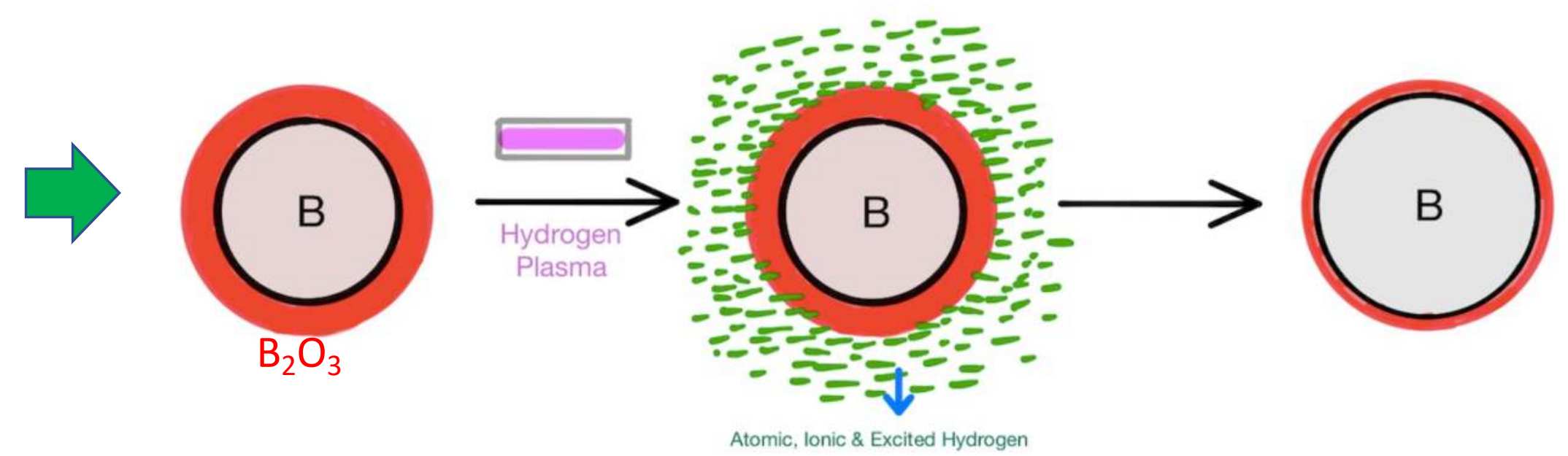




\section{Surface modification for passivation \& forming stable dispersions in fuels}

\section{Capping with Octyloxy groups}

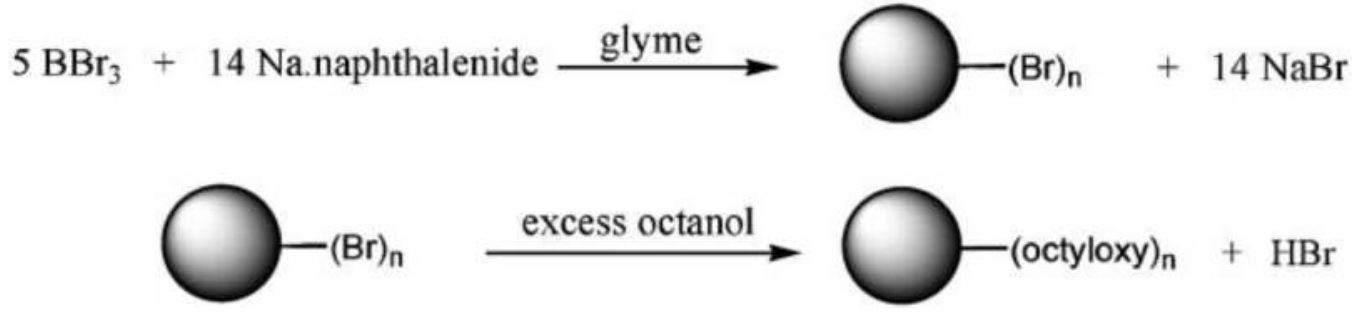

Pickering et al., RSC ChemComm, 2007

Capping with Silane

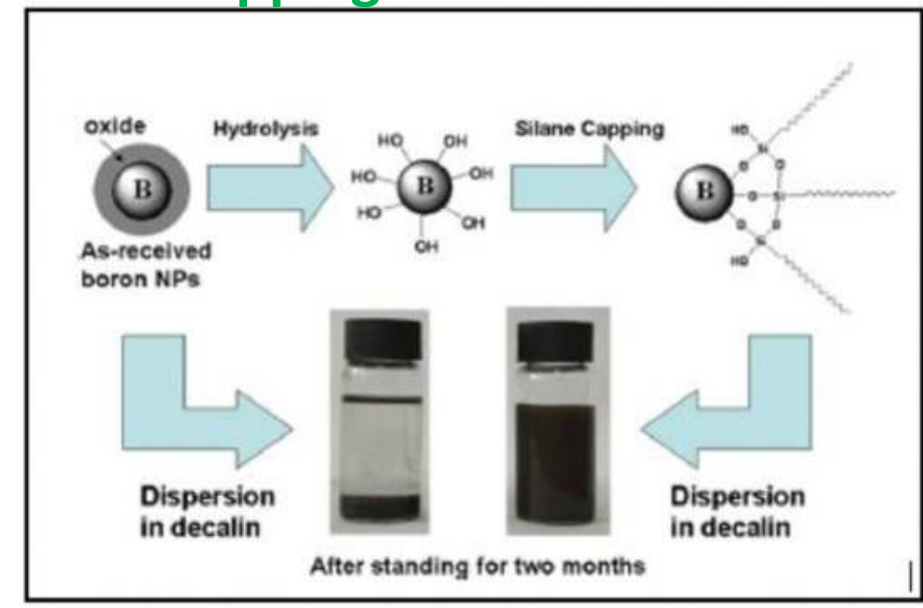

Meijie et al., Fuels, 2017

\section{Surface Functionalization using Halogens}

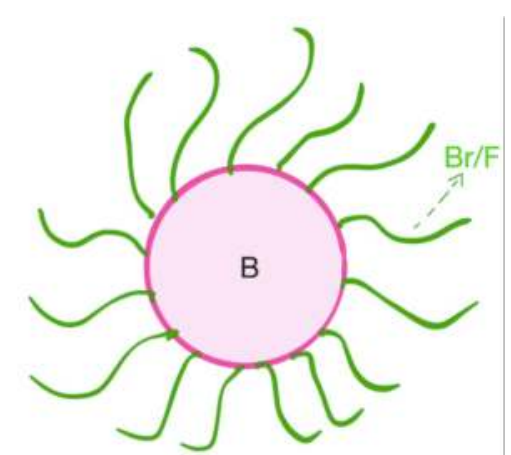

Bellott et al., RSC ChemComm, 2009

High energy Milling using oleic acid

Anderson et al., Energy \& Fuels, 2009

\section{Problems}

Longer processing/separation

times/achieving both passivation \&

stable dispersion 


\section{Both passivation and stable dispersions can be achieved by method developed by our lab}

\section{Plasma Enhanced Chemical Vapor Deposition (PECVD)}
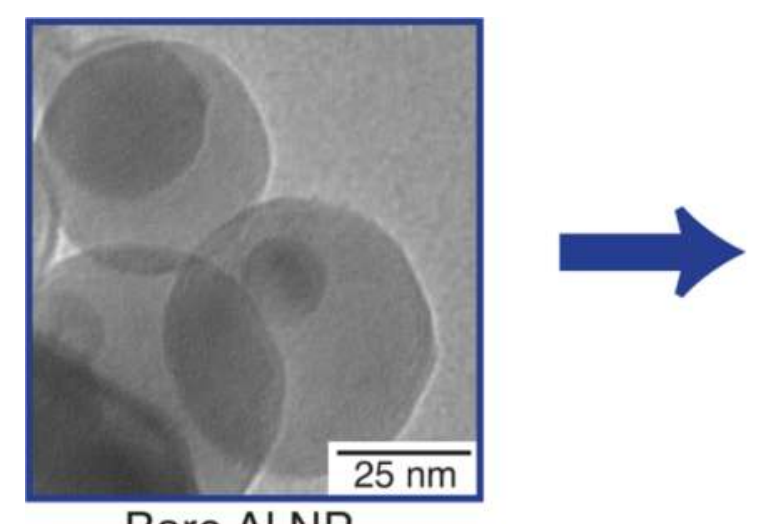

Bare AI NP

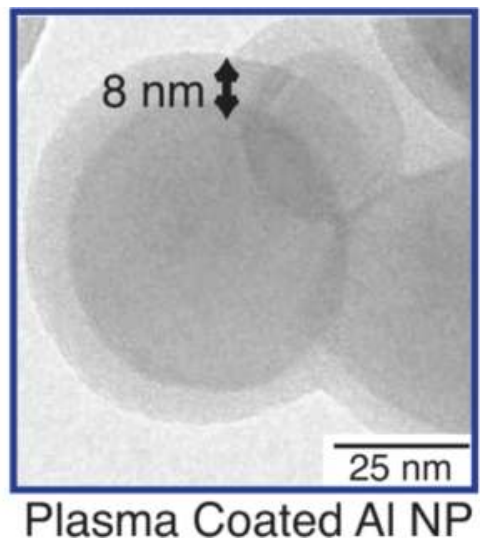

- Tailoring of coating chemistry

- Nanometer level control on coating thickness
Perfluoro decalin (PFD)

Better Coating Precursor!

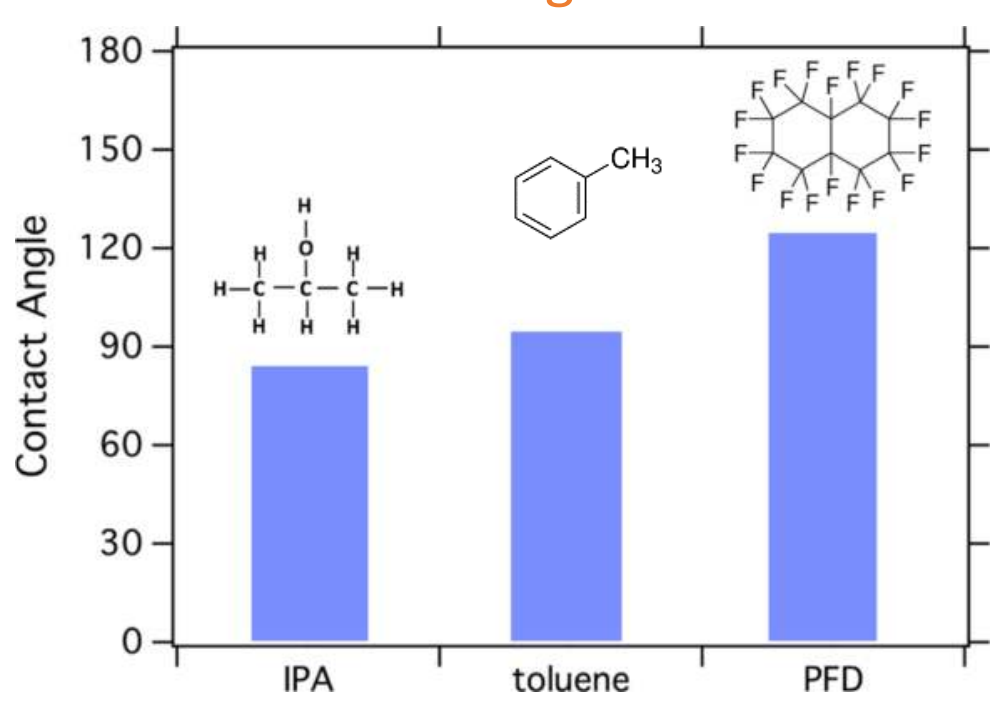

- Water Contact Angle: $125^{\circ}$

- Passivation against air \& humidity

- Can provide Improved dispersibility in liquid fuel 


\section{Goal - To combine HP reduction and PECVD passivation to produce energetically enhanced BNP}

- To reduce native oxide

- To form a passivation barrier against air \& moisture

- To form stable dispersions in jet propellants

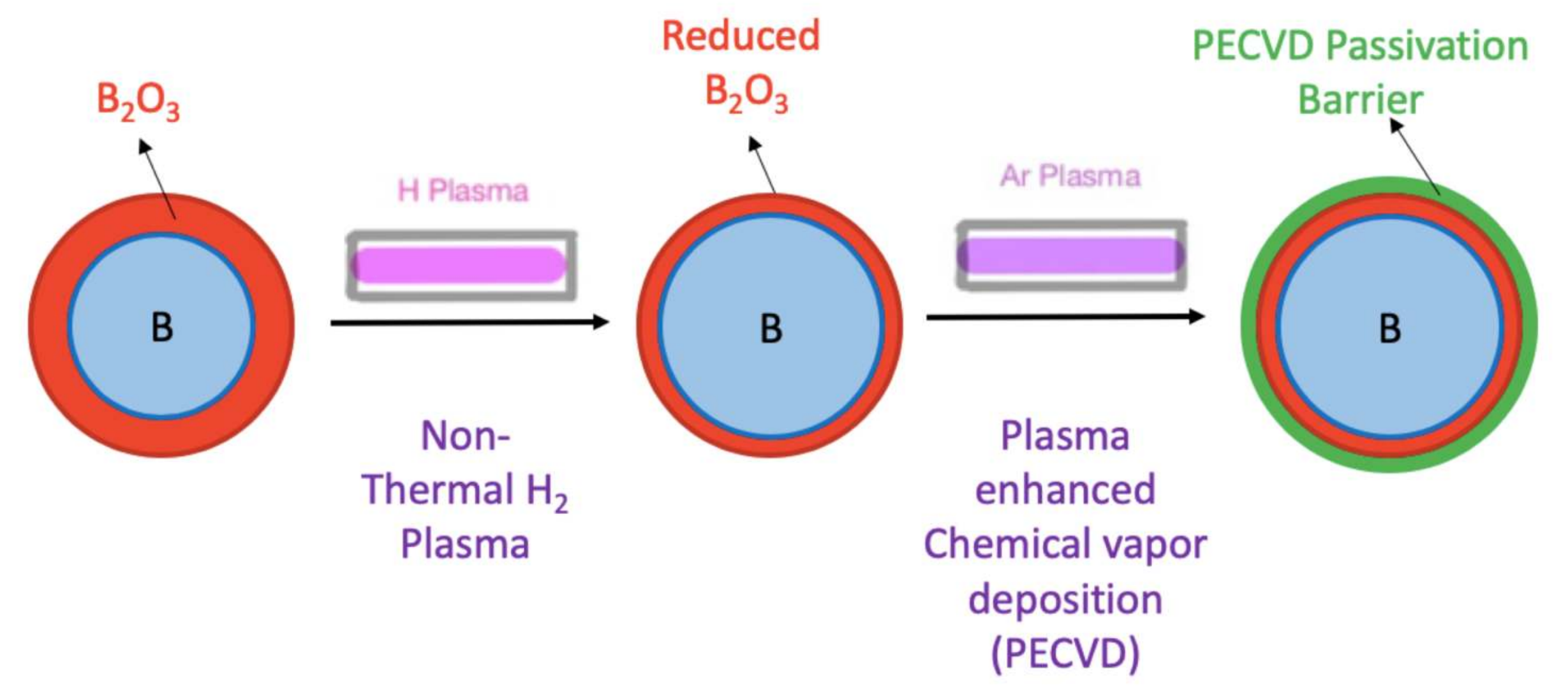




\section{Non-thermal plasma experimental setup}

\section{Components}

- Vacuum Pump with LN trap

- RF generator with matching box

- Tubular Reactor

- Magnetic Stirrer

- Hydrogen Transport System

- Argon assisted precursor delivery system

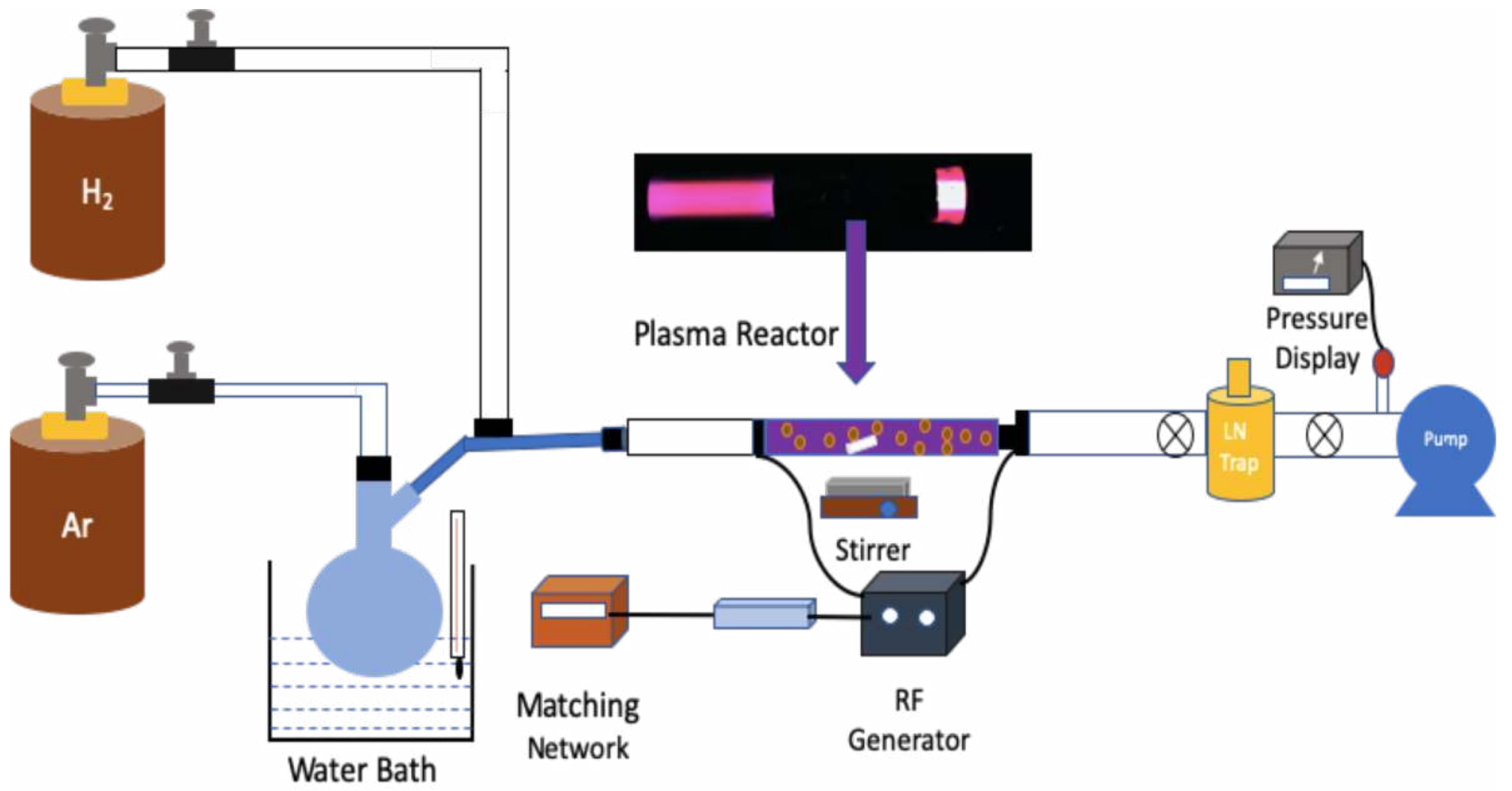




\section{Experimental Methods}

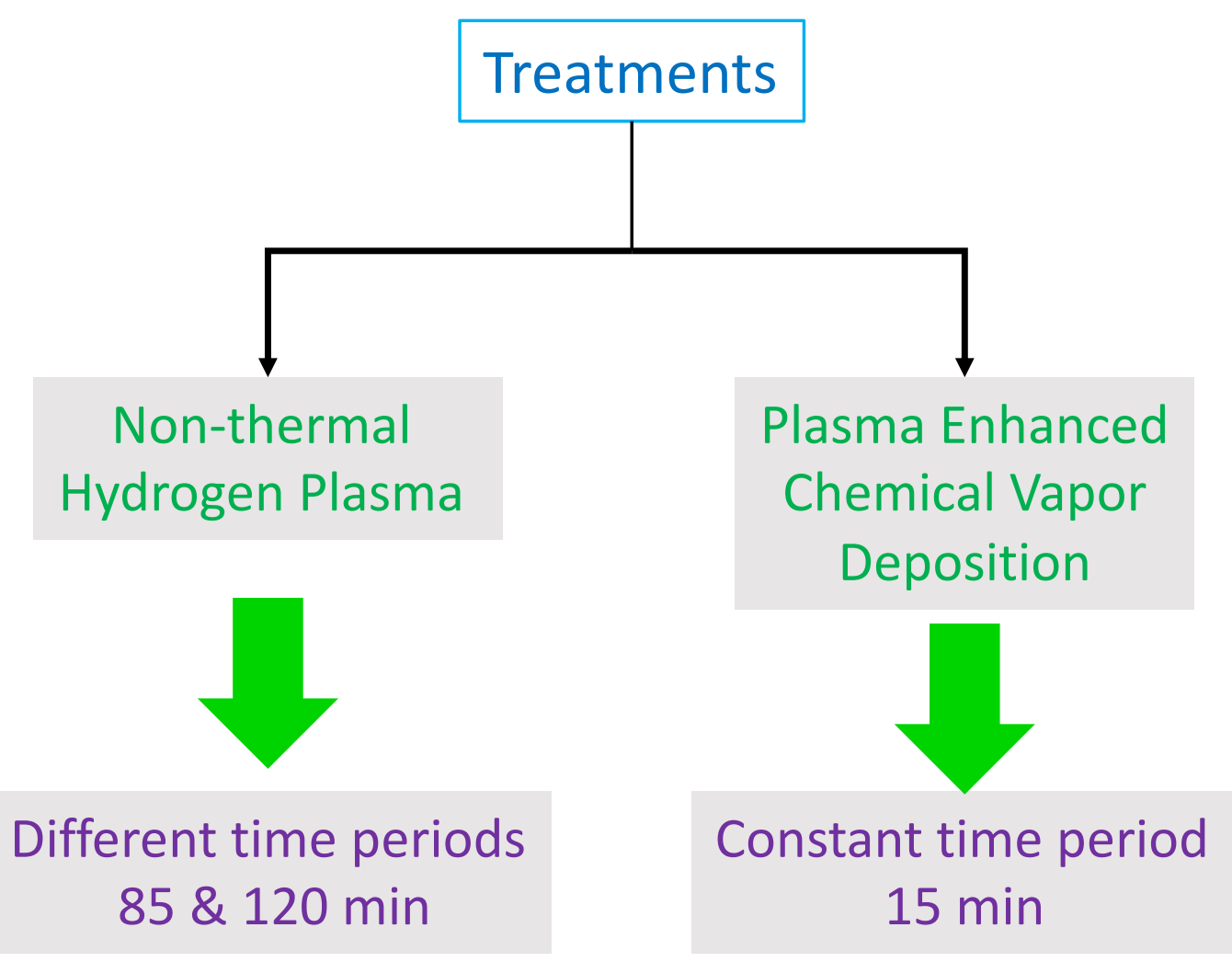

Average Particle Size

BNP: $70 \mathrm{~nm}$

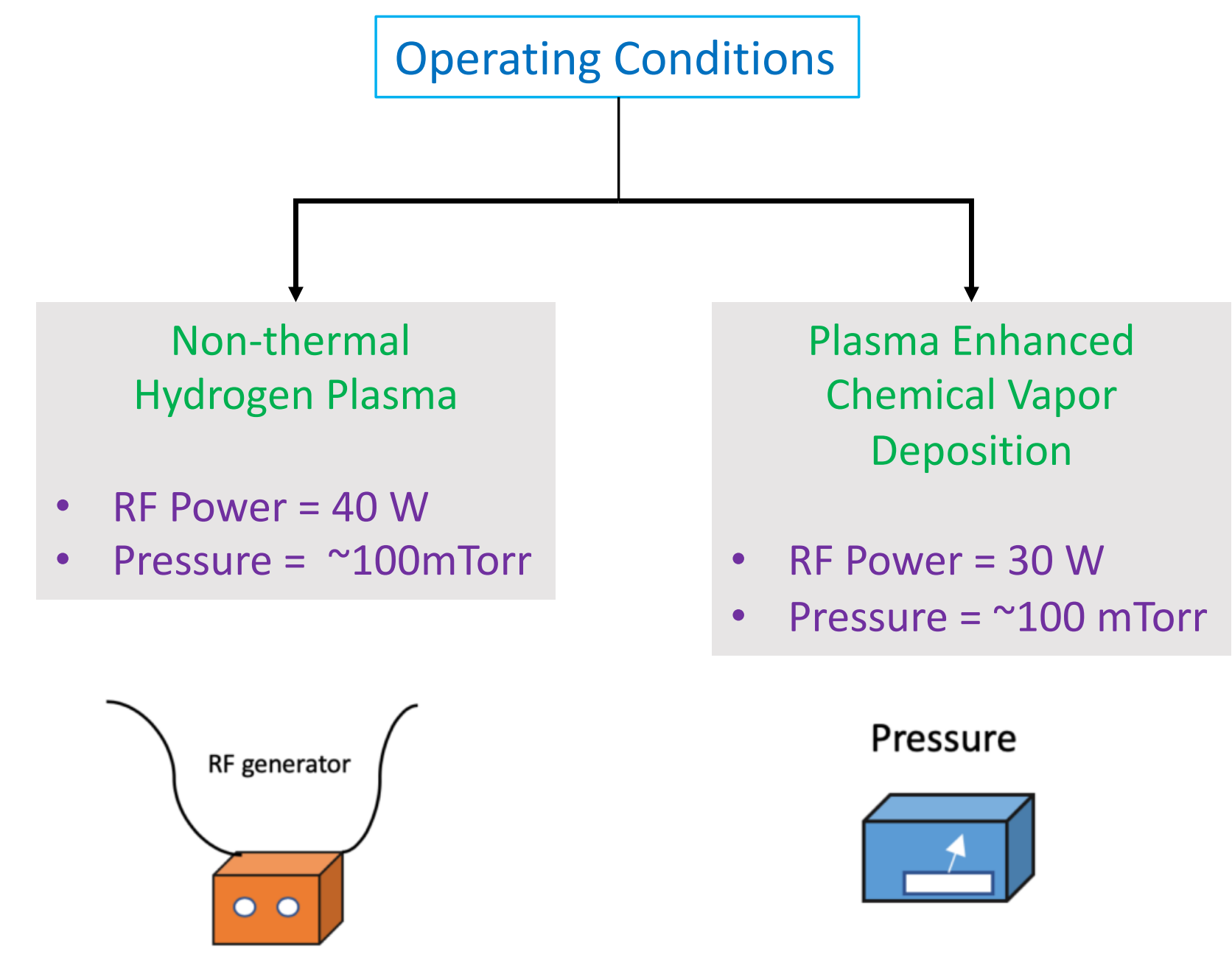




\section{Characterization methods to get reduction, passivation \& energy evidences}
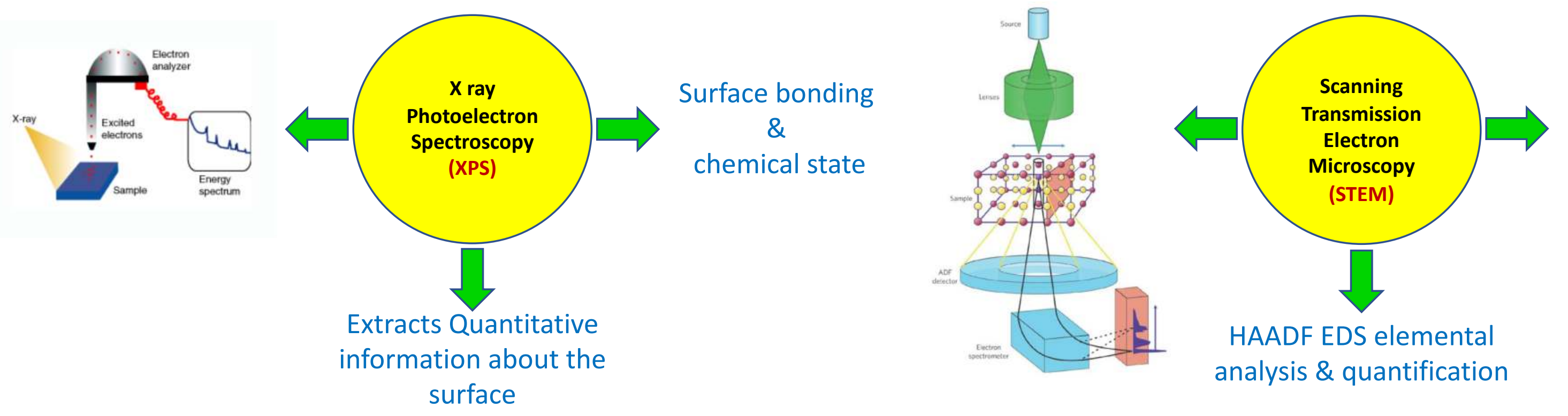

High

Resolution

image

evidence

DSC : Differential Scanning Calorimetry
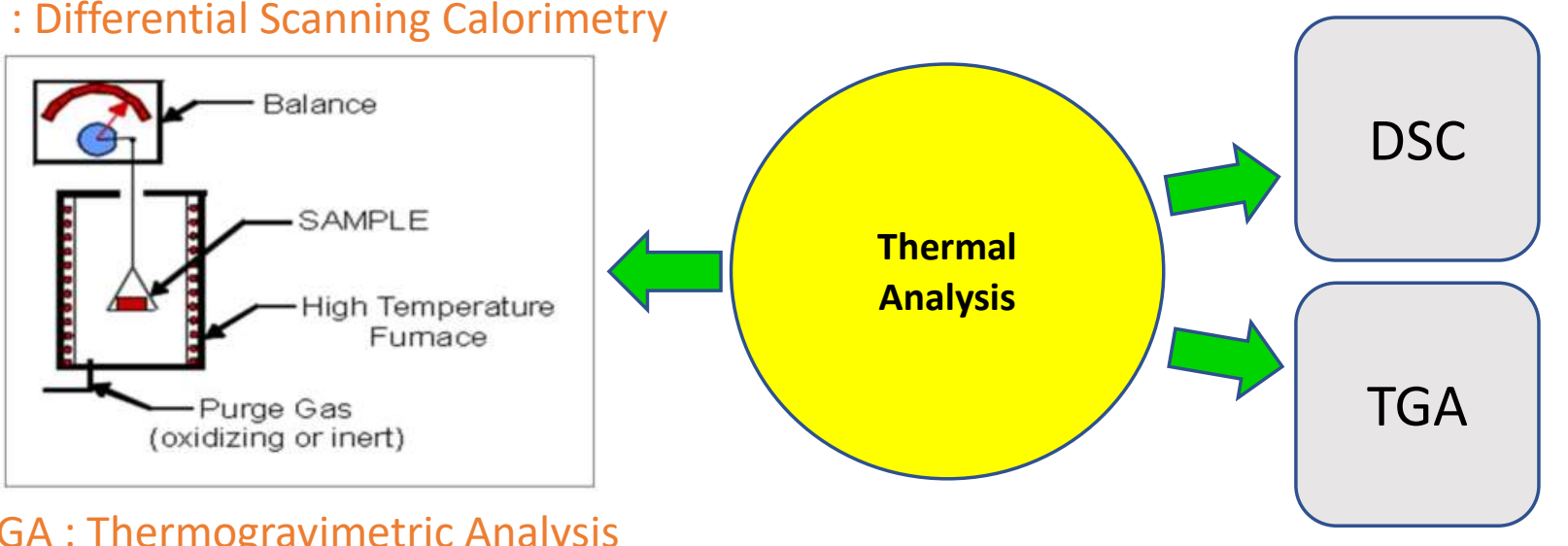

Measures Heat flow

Oxidation Kinetics \& Weight change 
High Resolution TEM images give evidences about thickness of oxide layer \& coating

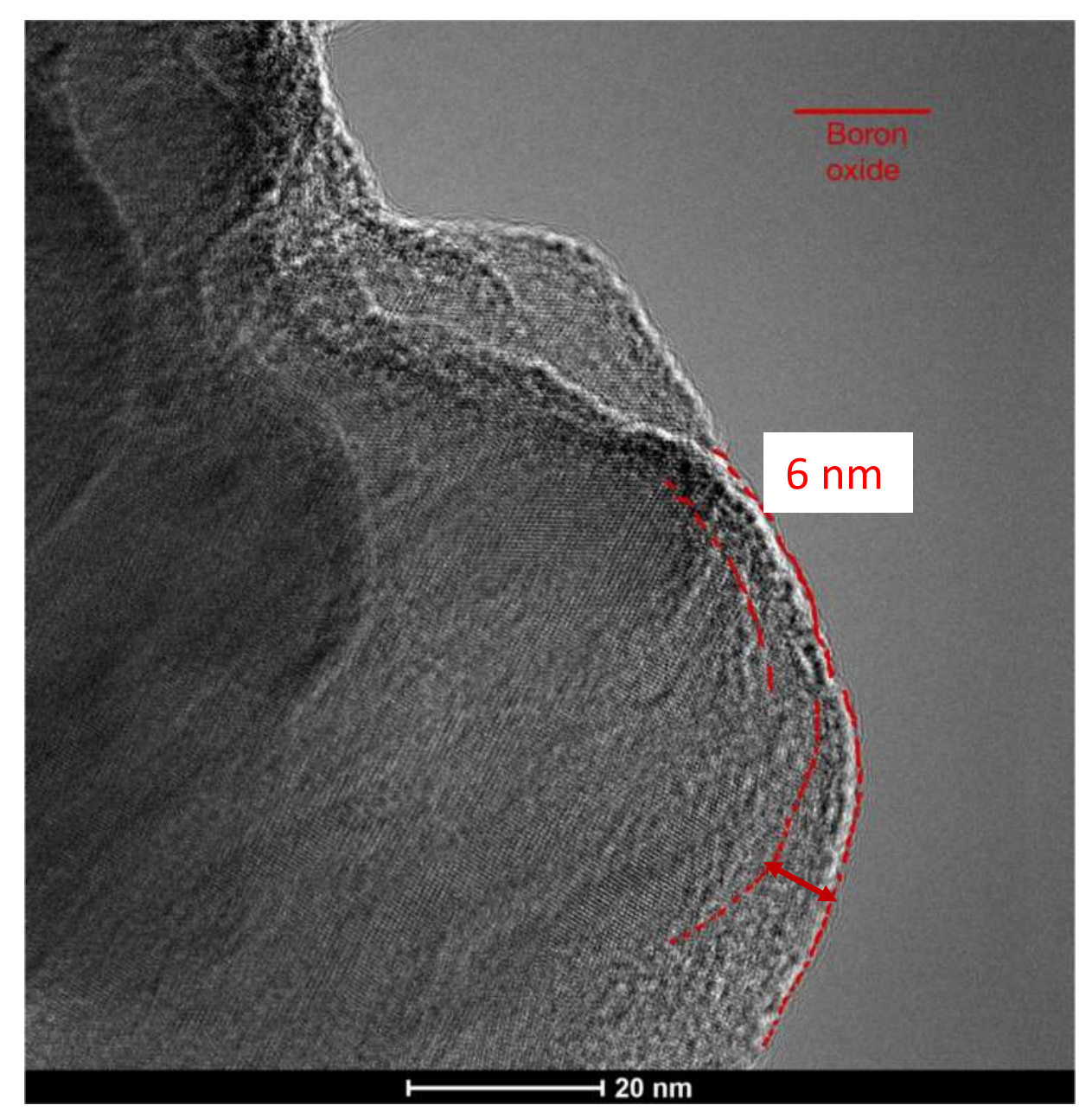

Untreated BNP

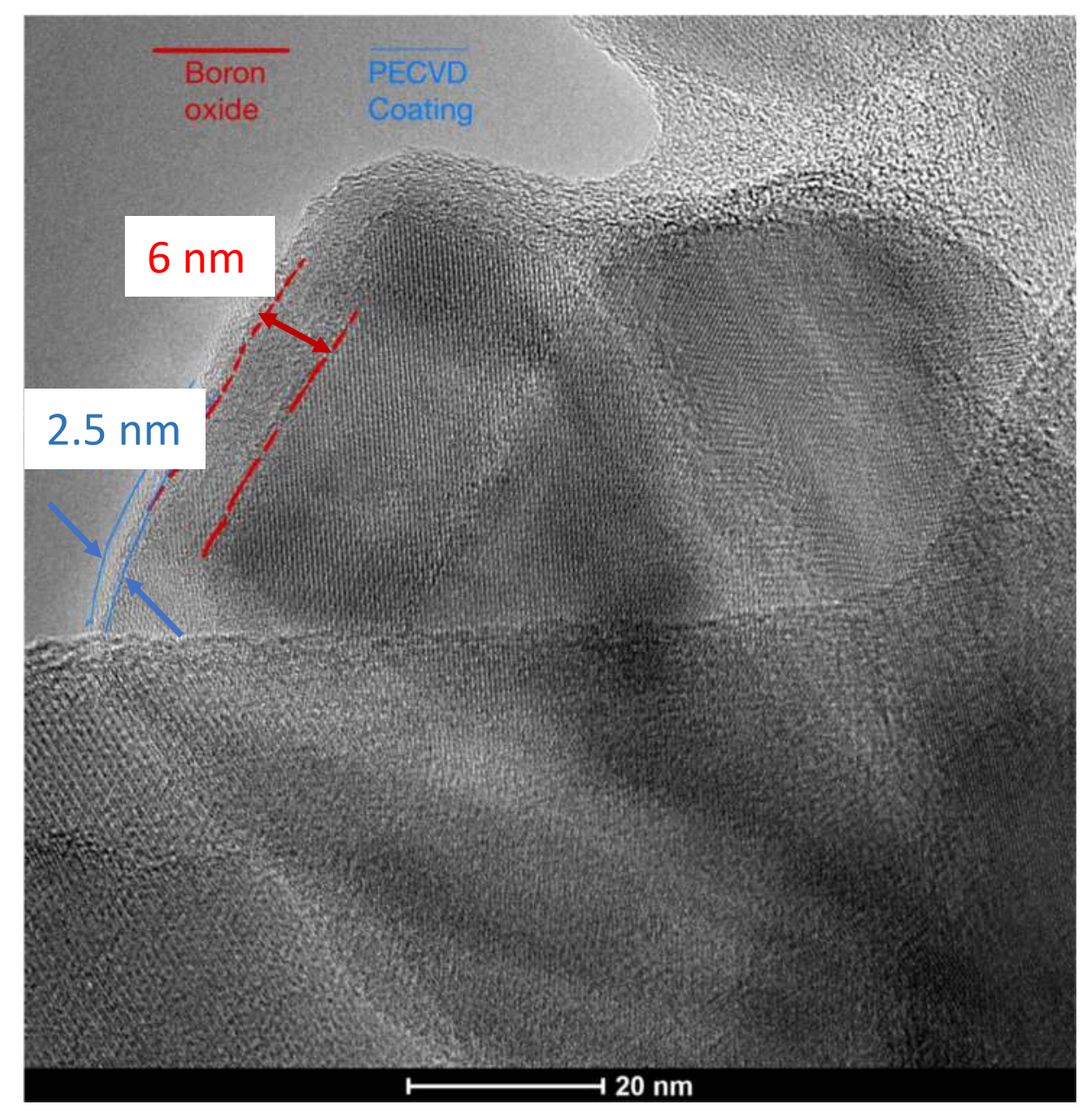

PECVD (15) treated BNP 
High Resolution TEM images suggested reduction of native oxide layer

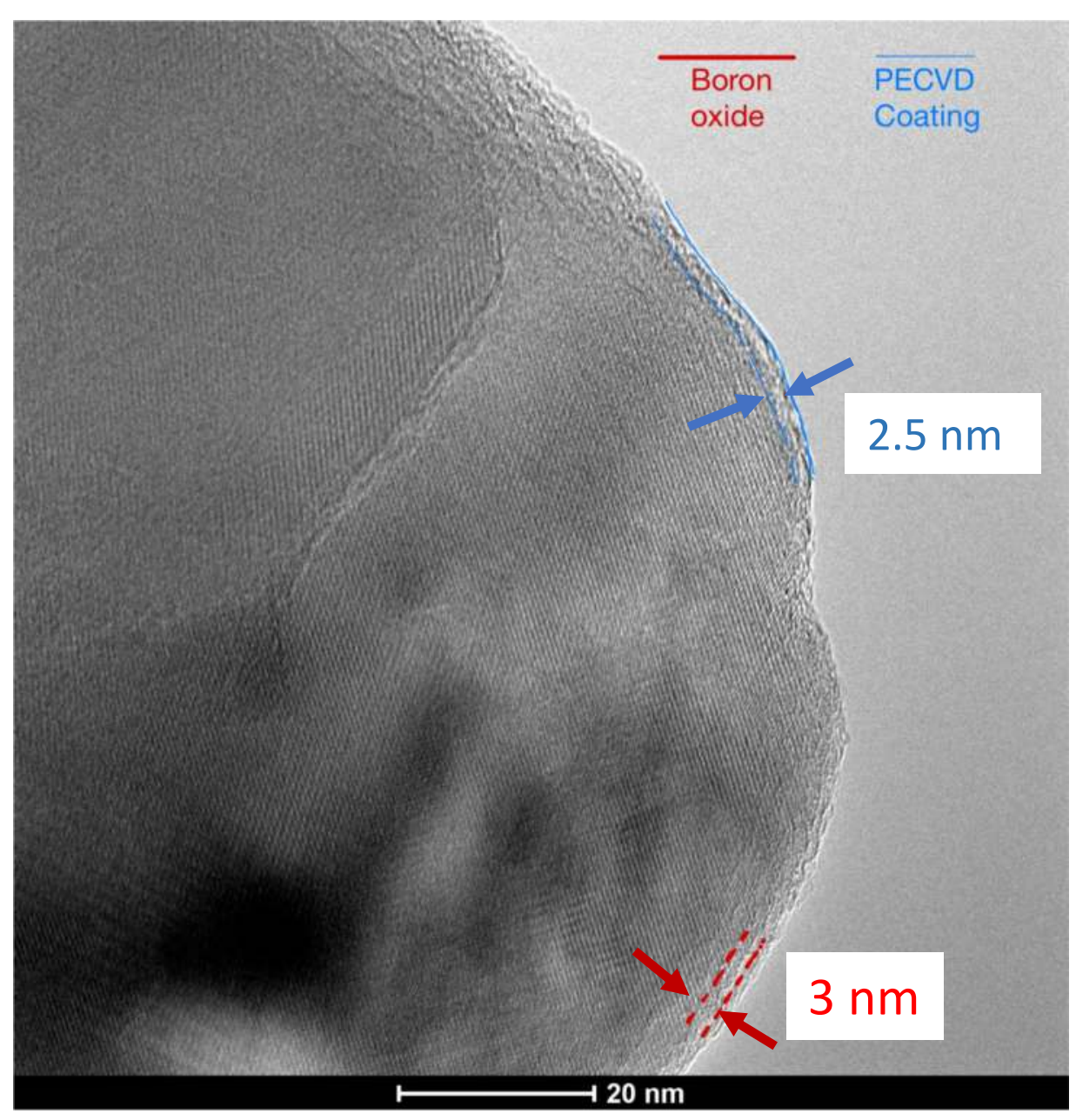

HP(85) PECVD(15)

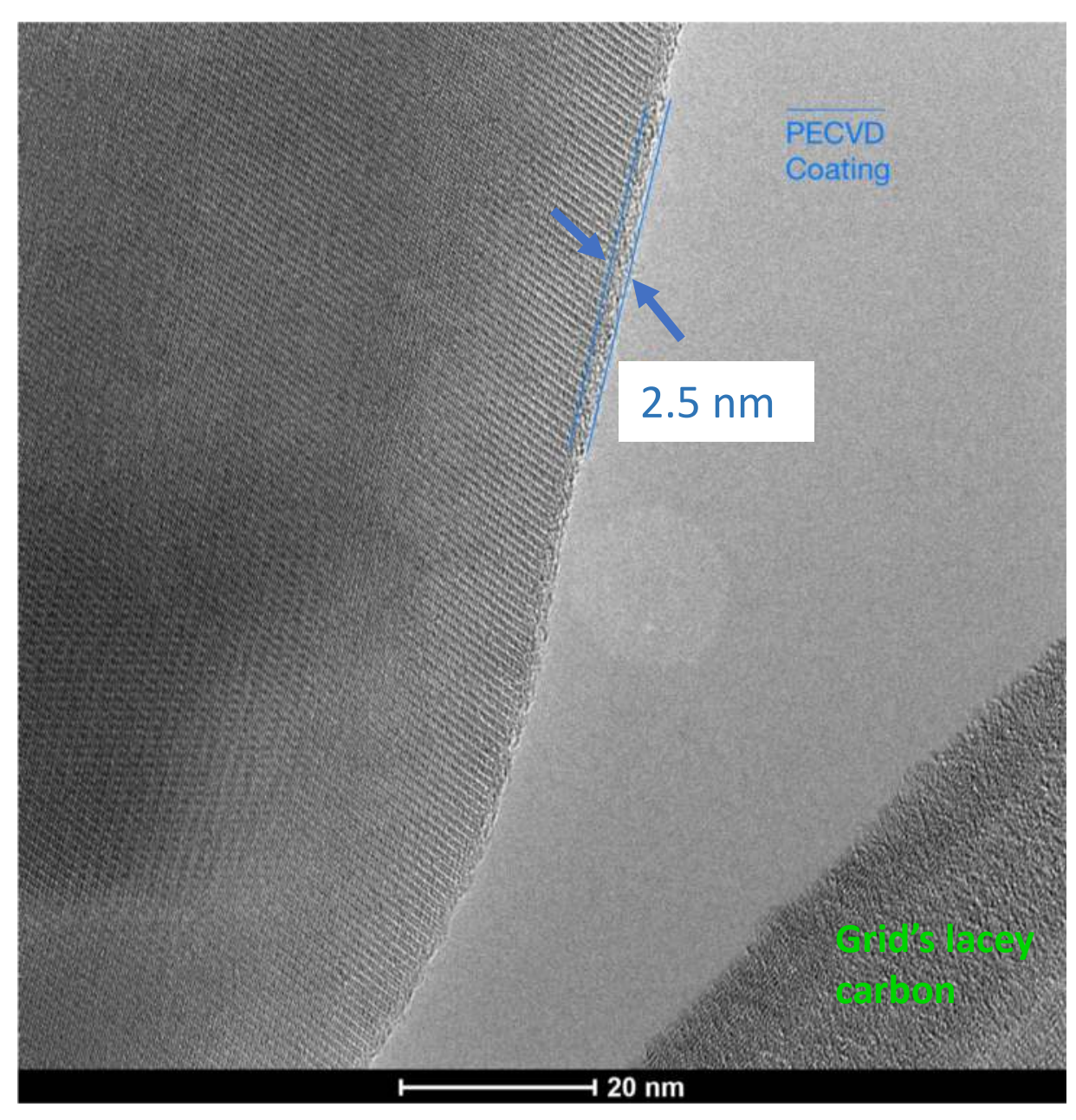

HP(120) PECVD(15) 


\section{STEM-EDS in HAADF shows evidence of reduction of atomic} concentration of oxygen through elemental analysis
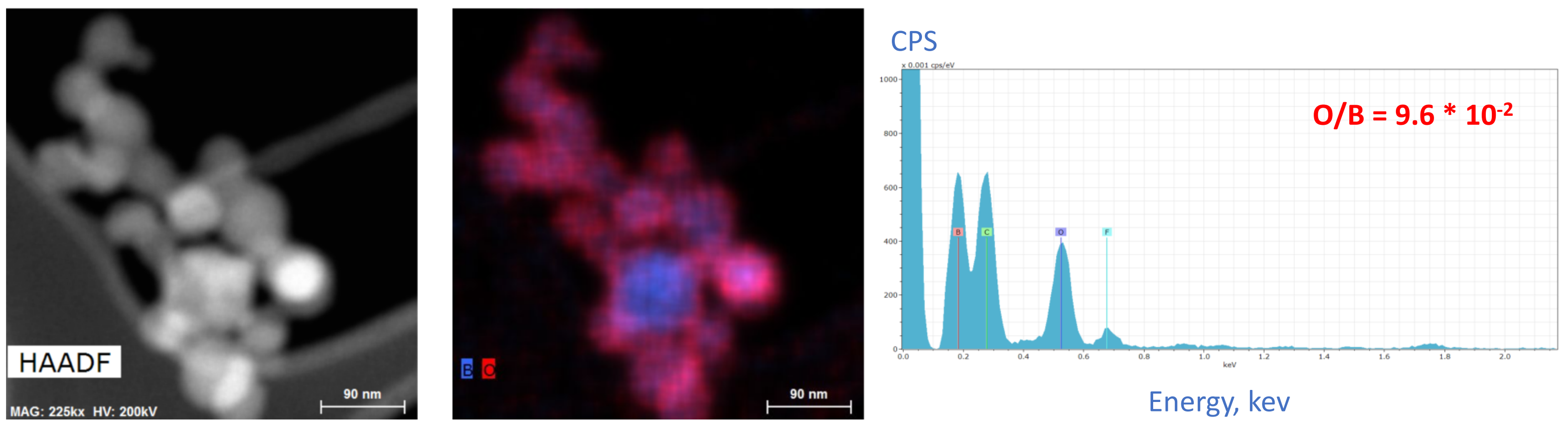

Quantitative Analysis is done by calculating

Without HP treatment relative atomic composition of Oxygen to Boron (O/B) 


\section{Summary of HAADF EDS Results}

\begin{tabular}{|c|c|}
\hline $\begin{array}{l}\text { Treatments on Boron } \\
\text { Nanoparticles } \\
\text { (Time is shown in } \\
\text { brackets in minutes) }\end{array}$ & O/B * $10^{-2}$ \\
\hline Untreated Sample & 9.6 \\
\hline HP (85) PECVD (15) & 2.5 \\
\hline HP (120) PECVD (15) & 0.68 \\
\hline
\end{tabular}

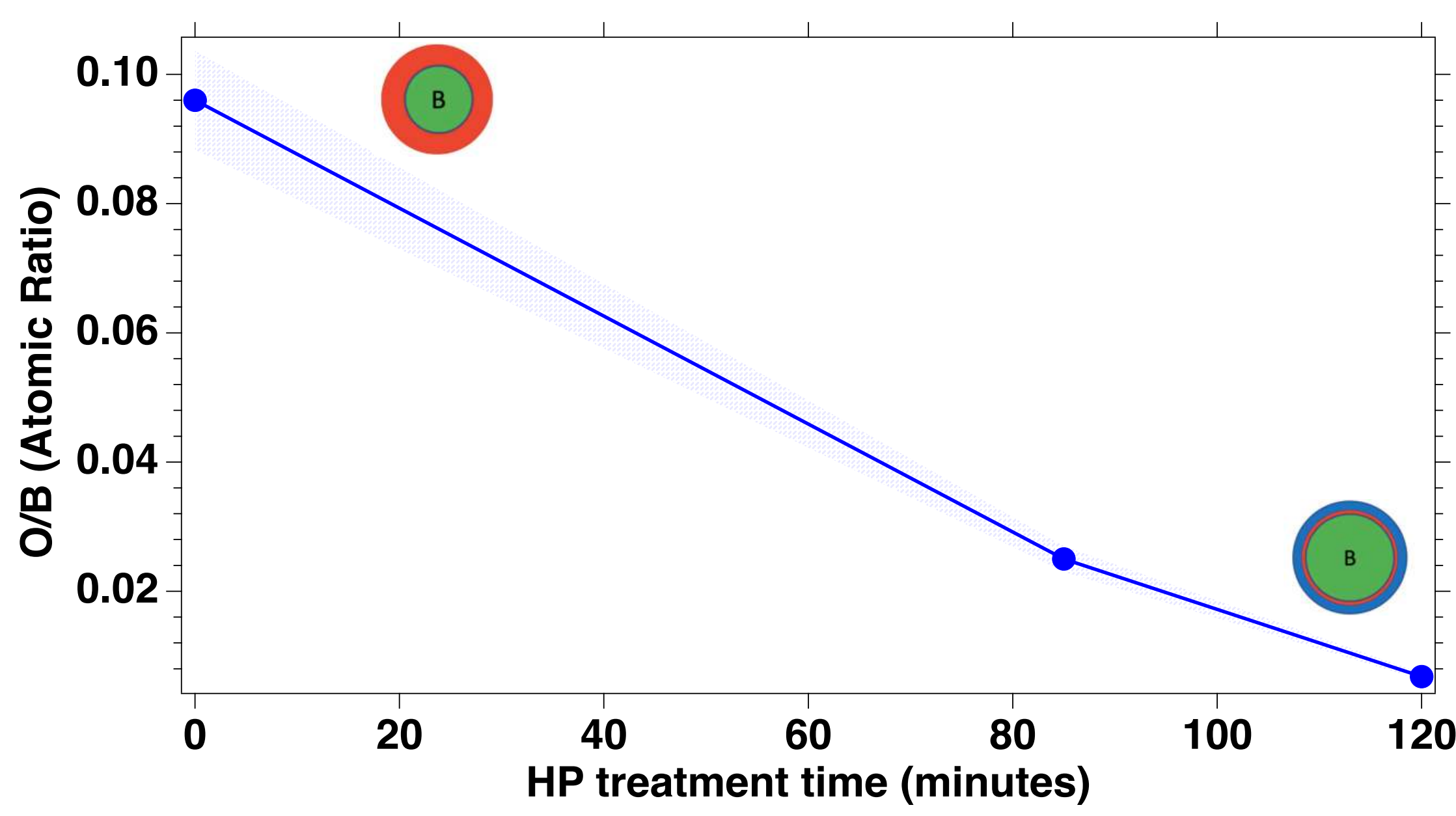


High Resolution XPS is done to extract quantitative information on the surface

\section{Sampling Depth: 10-15 nm}
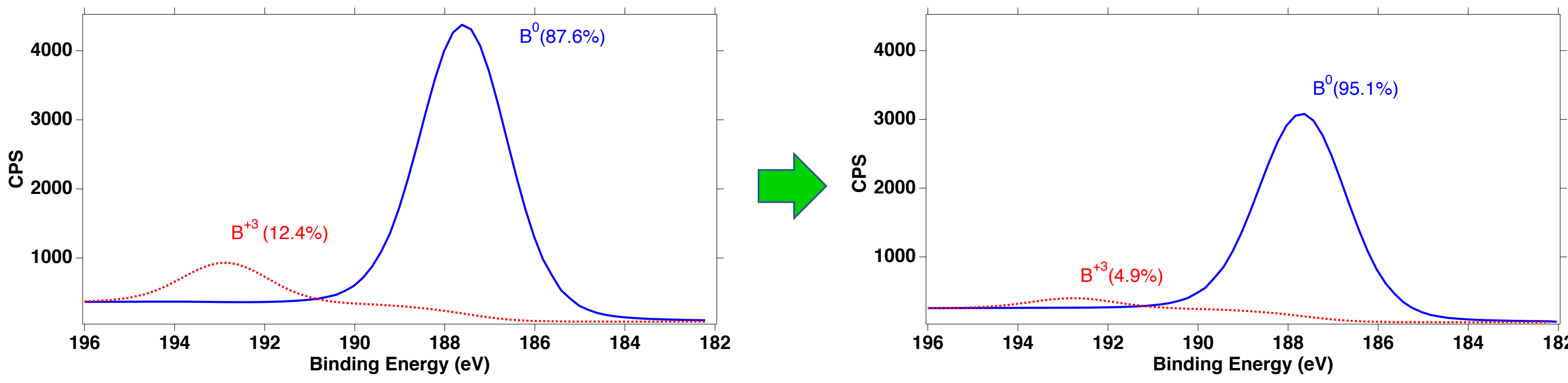

Untreated BNP; $\mathrm{B}^{+3}=12.4 \%$

HP(120) PECVD(15) ; $\mathrm{B}^{+3}=4.9 \%$ 


\section{Summary of XPS Results}

\begin{tabular}{|c|c|}
$\begin{array}{c}\text { Treatments on Boron Nanoparticles } \\
\text { (Time is shown in brackets in } \\
\text { minutes) }\end{array}$ & $\mathrm{B}^{+3}$ Concentration (\%) \\
\hline Untreated Sample & 12.74 \\
\hline HP (0) PECVD (15) & 12.43 \\
\hline HP (85) PECVD (15) & 8.79 \\
\hline HP (120) PECVD (15) & 4.99 \\
\hline
\end{tabular}

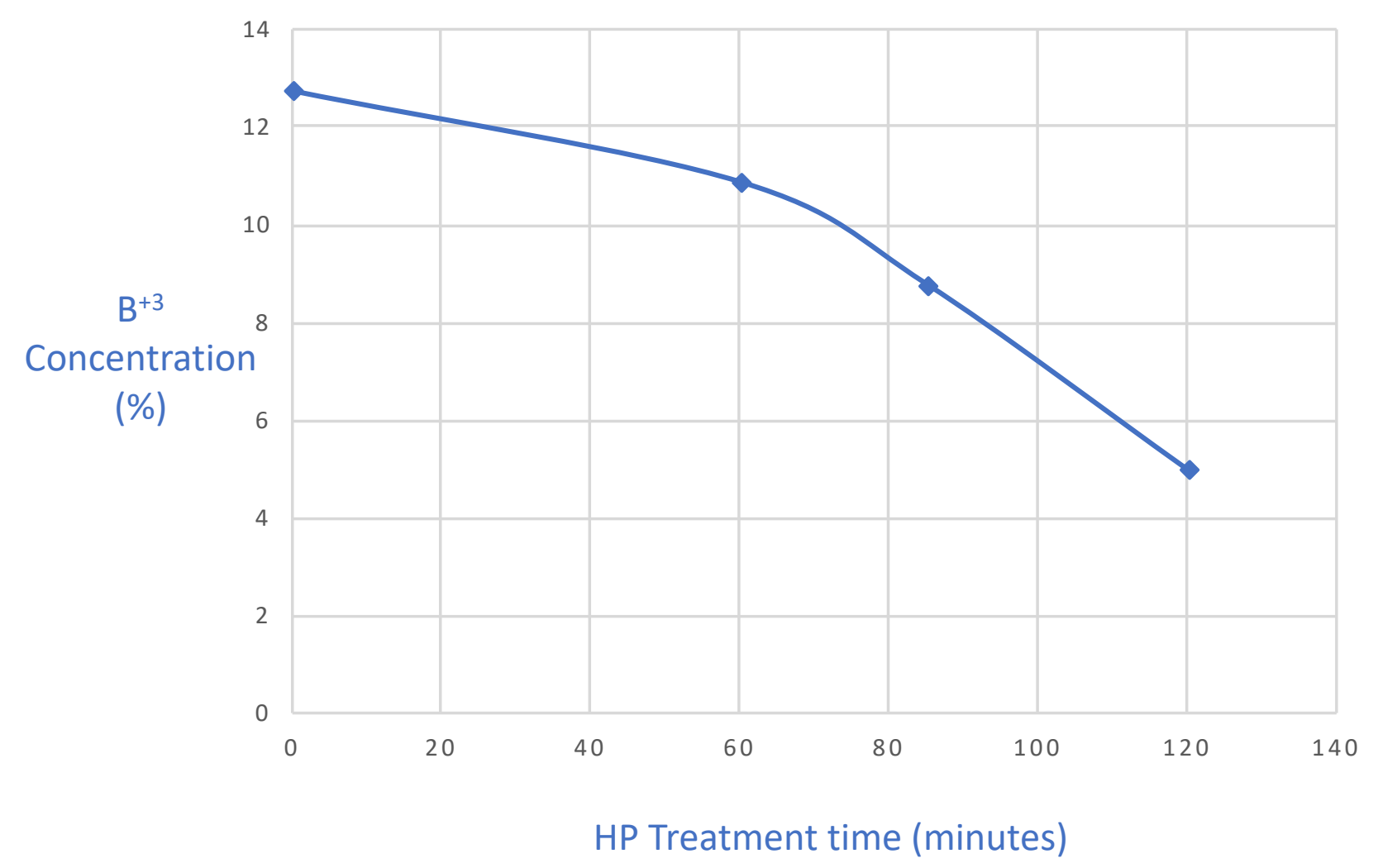




\section{Weight change of untreated \& plasma treated BNPs were measured \& compared using TGA}

\section{Simultaneous DSC|TGA}

- Heating Rate: $30^{\circ} \mathrm{C} / \mathrm{min}$ from $\sim 25^{\circ} \mathrm{C}$

\section{Method} to $1400^{\circ} \mathrm{C}$

- Oxidizer used: Air (100ml/min)

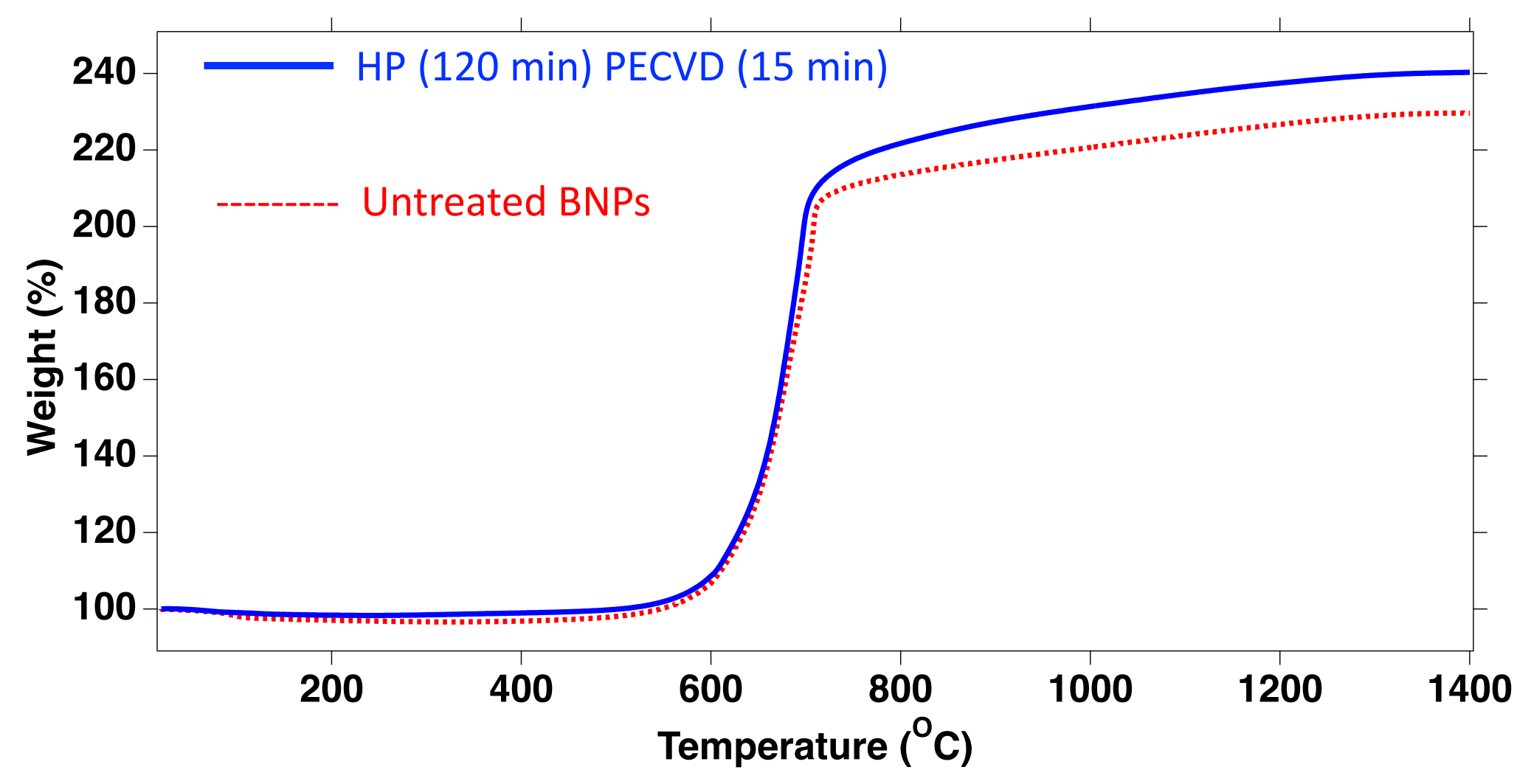


Gravimetric Energy density of untreated \& plasma treated BNPs were measured \& compared using DSC

Simultaneous DSC|TGA

- Heating Rate: $30^{\circ} \mathrm{C} / \mathrm{min}$ from $\sim 25^{\circ} \mathrm{C}$ to $1400^{\circ} \mathrm{C}$

- Oxidizer used: Air (100ml/min)

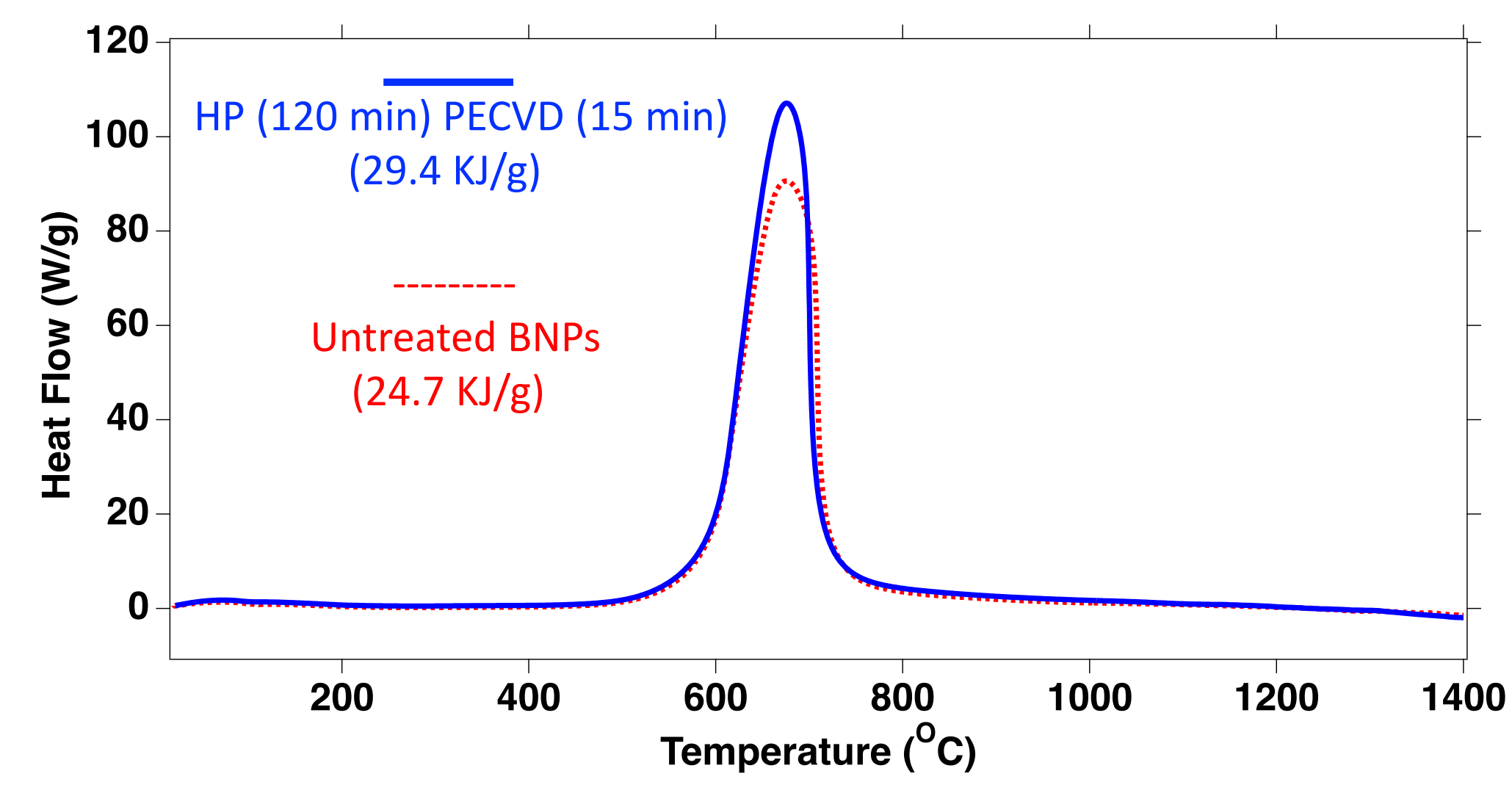




\section{Summary of DSC Results}

\begin{tabular}{|c|c|c|}
\hline $\begin{array}{c}\text { Treatments on Boron } \\
\text { Nanoparticles } \\
\text { (Time is shown in } \\
\text { brackets in minutes) }\end{array}$ & $\begin{array}{c}\text { Enthalpy of } \\
\text { Combustion }(\mathrm{KJ} / \mathrm{g}) \\
\text { with error of }+/-2 \%\end{array}$ & $\begin{array}{c}\% \text { Increase with } \\
\text { respect to the } \\
\text { reference } \\
\text { (untreated BNP) }\end{array}$ \\
\hline Untreated Sample & 24.69 & $\begin{array}{c}0 \\
\text { (Reference) }\end{array}$ \\
\hline HP (0) PECVD (15) & 25.62 & +3.7 \\
\hline HP (85) PECVD (15) & 27.90 & +13 \\
\hline HP (120) PECVD (15) & 29.42 & +19 \\
\hline
\end{tabular}

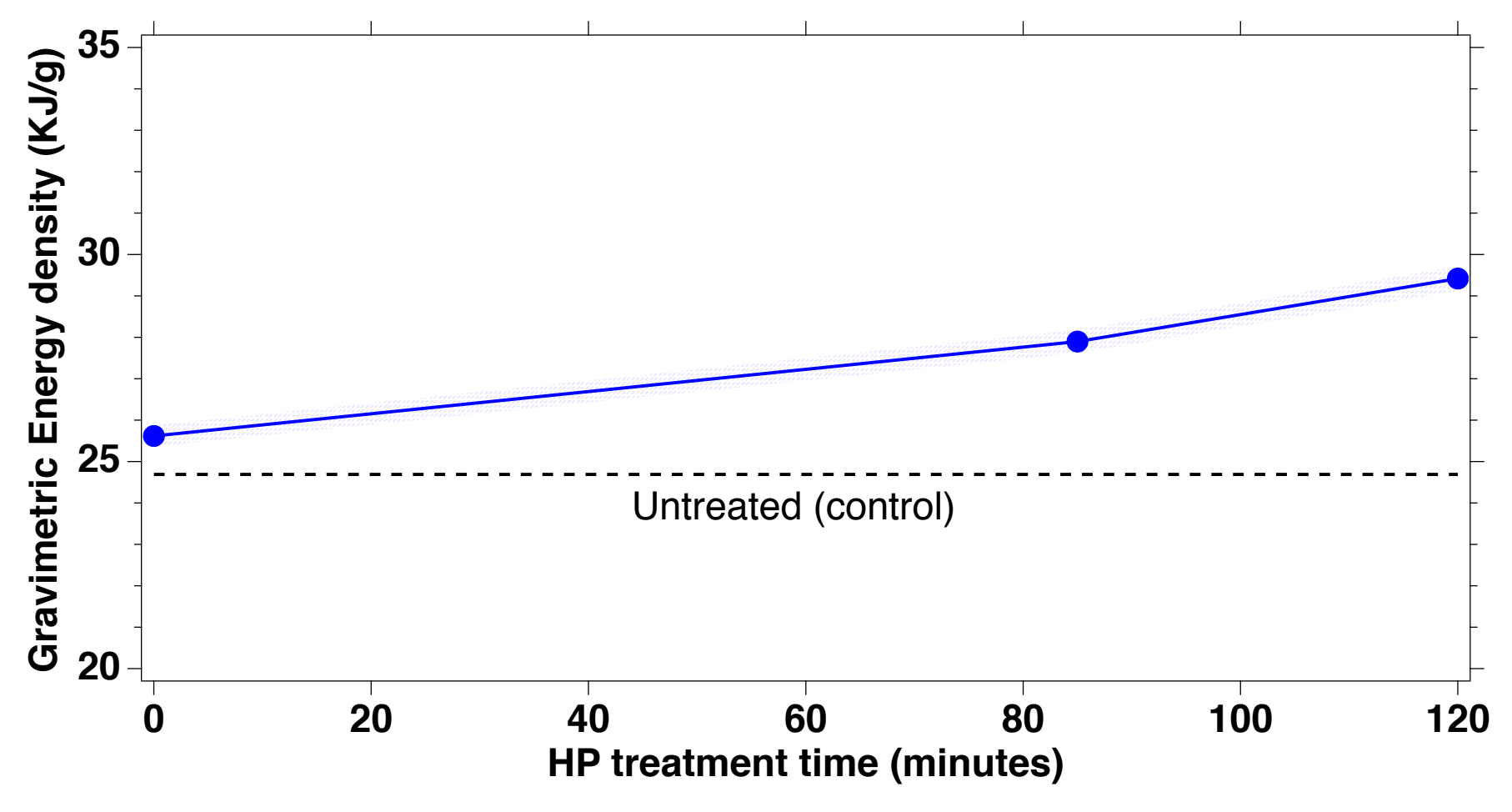




\section{Summary}

- Developed unique in-situ method of non-thermal plasma reduction + passivation of BNP

- Significant reduction in native oxide layer (diffusion barrier for oxidizer)

- Formation of passivation barrier which is preventing re-oxidation for at least 2 months

- Enhanced gravimetric energy density of BNP as a result of oxide layer reduction
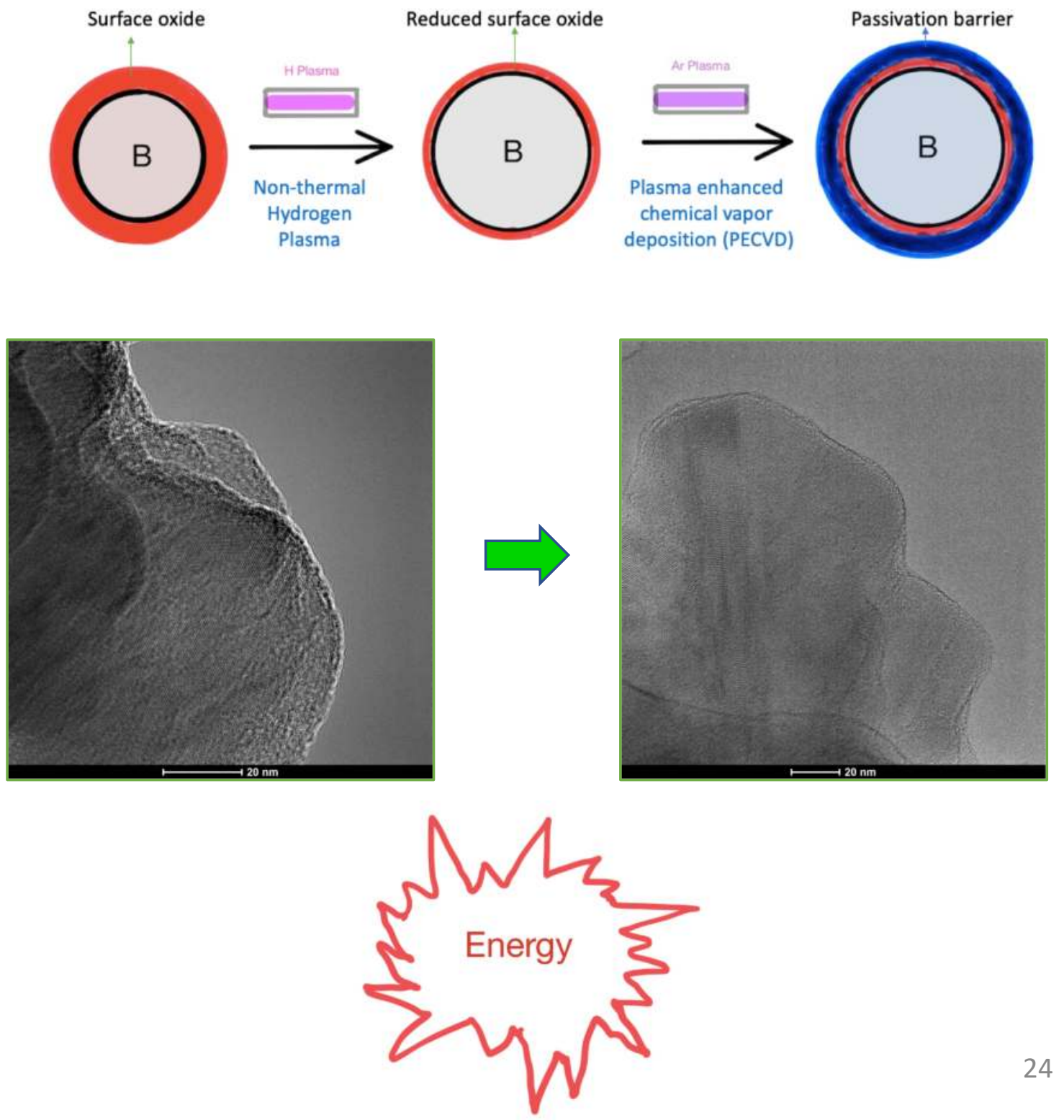
Acknowledgements

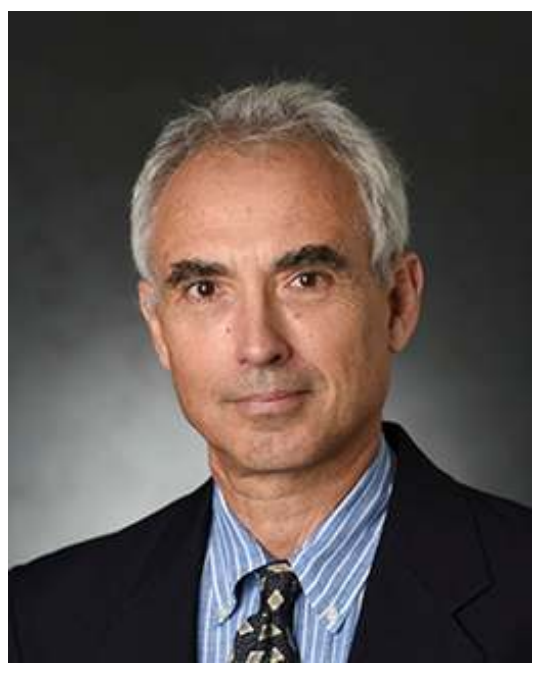

Dr. Themis Matsoukas

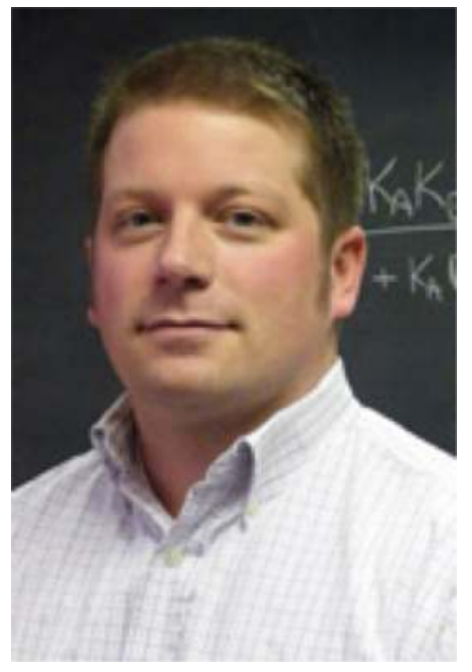

Dr. Rob Rioux

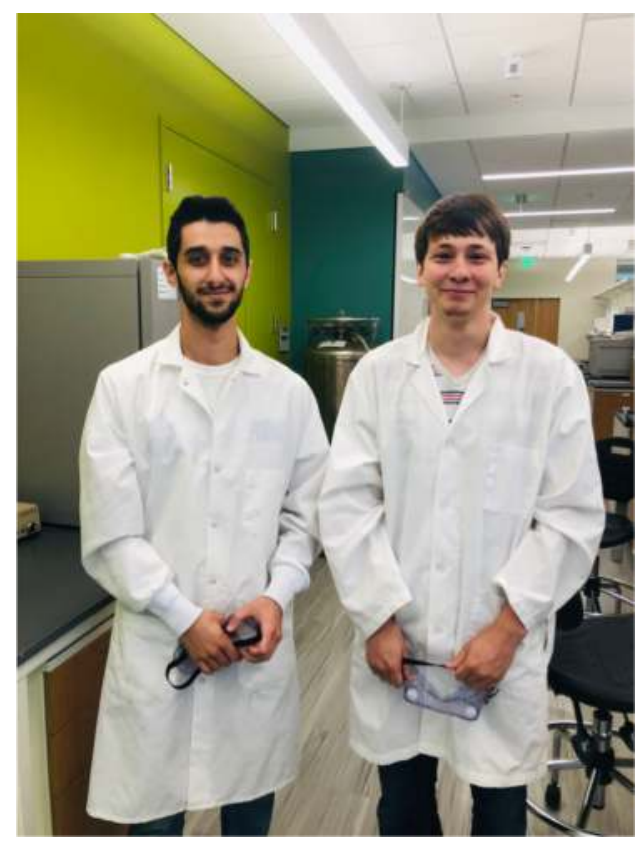

Toni \& Troy (TT)

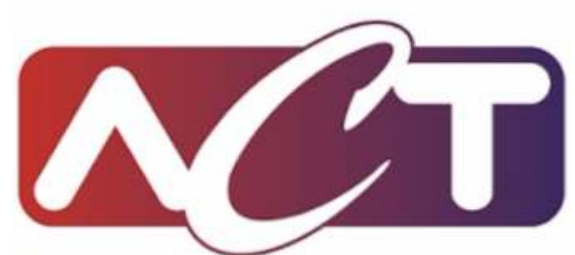

ADVANCED COOLING TECHNOLOGIES The Thermal Management Experts | www.1-ACT.com

PennState

Institute

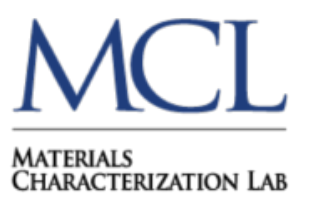

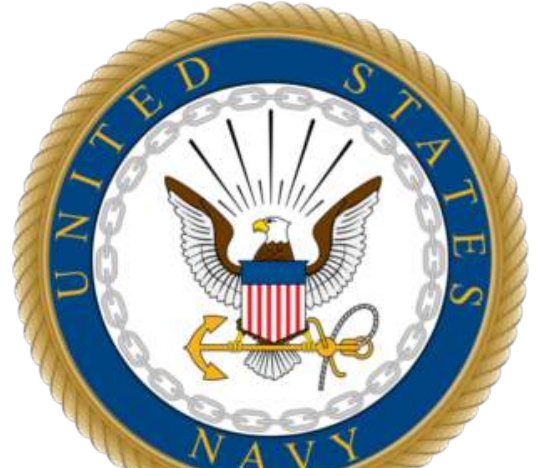

